University of Nebraska - Lincoln

DigitalCommons@University of Nebraska - Lincoln

1983

\title{
Thermal Propagation and Stability in Superconducting Films
}

Kenneth E. Gray

Argonne National Laboratory

Robert T. Kampwirth

Argonne National Laboratory

John F. Zasadzinski

Argonne National Laboratory

Stephen P. Ducharme

Argonne National Laboratory, sducharme1@unl.edu

Follow this and additional works at: https://digitalcommons.unl.edu/physicsducharme

Part of the Condensed Matter Physics Commons

Gray, Kenneth E.; Kampwirth, Robert T.; Zasadzinski, John F.; and Ducharme, Stephen P., "Thermal Propagation and Stability in Superconducting Films" (1983). Stephen Ducharme Publications. 57. https://digitalcommons.unl.edu/physicsducharme/57

This Article is brought to you for free and open access by the Research Papers in Physics and Astronomy at DigitalCommons@University of Nebraska - Lincoln. It has been accepted for inclusion in Stephen Ducharme Publications by an authorized administrator of DigitalCommons@University of Nebraska - Lincoln. 


\title{
Thermal propagation and stability in superconducting films
}

\author{
K E Gray, R T Kampwirth, J F Zasadzinski and S P Ducharme $\dagger$ \\ Solid State Science Division, Argonne National Laboratory, Argonne, IL 60439, USA
}

Received 8 March 1982, in final form 23 July 1982

\begin{abstract}
Thermal propagation and stable hot spots (normal domains) are studied in various high $T_{\mathrm{c}}$ superconducting films ( $\mathrm{Nb}_{3} \mathrm{Sn}, \mathrm{Nb}, \mathrm{NbN}$ and $\mathrm{Nb}_{3} \mathrm{Ge}$ ). The prediction of the thermal propagation velocity of the long-standing model of Broom and Rhoderick is verified quantitatively in the regime of its validity. A new energy balance model is shown to give reasonable quantitative agreement of the dependence of the propagation velocity on the length of short normal domains. The steady state (zero velocity) measurements indicate the existence of two distinct situations for films on high thermal conductivity (sapphire) substrates. For low power per unit area the film and substrate have the same temperature, and the thermal properties of the substrate dominate. However, for higher power densities in short hot spots, the coupling is relatively weak and the thermal properties of the film alone are important. Here a connection is made between the critical current stability of superconducting films and a critical hot spot size for thermal propagation. As a result efficient heat removal is shown to dominate the stabilisation of superconducting films. The strong and weak coupling situations also lead to modifications of the models for propagation velocities on sapphire substrates. These models are used to explain quantitatively self-healing of hot spots and a curious switching phenomena in a superconducting film in which a hot spot grows and collapses repeatedly over a very narrow range of current. Finally there is a discussion of the potential use of the thermal propagation model in applications of superconductors, especially switches.
\end{abstract}

\section{Introduction}

There are several reasons for studying the thermal propagation velocity (Bremer and Newhouse 1958) of a normal-superconductor (NS) boundary in current-carrying superconducting films. The first is to understand better the stabilisation of the critical current density $J_{\mathfrak{c}}$. Secondly there are long-standing theoretical models (Broom and Rhoderick 1960, to be referred to as BR, Newhouse 1964) for the propagation velocity which have not been verified quantitatively. Additionally, in certain applications it is of interest to evaluate the potential of thermal propagation to provide protection from fusing at hot spots. Finally in some switching applications, thermal propagation from a limited number of intentional hot spots may be practical to complete switching of the superconducting element (Gray et al 1980).

\subsection{Stability of critical current density}

There have been several studies (Wipf and Martinelli 1973, Wilson and Iwasa 1978, Wipf 1979) aimed at relating $J_{c}$ of bulk superconductors to the concept of a minimum

$\dagger$ Present address: Department of Physics, University of Southern California, Los Angeles, CA 90007, USA. 
propagating zone (MPZ). The MPZ is the minimum length of a normal zone in a superconductor which will thermally propagate and thus quench superconductivity. As an example of the use of this concept in a large superconducting magnet, the MPZ determines constraints on mechanical movements of windings during operation, which dissipate a large amount of heat locally. Theoretically one attacks the heat flow equation and experimentally one heats a variable length of the superconducting winding above $T_{\mathrm{c}}$ and checks for breakdown.

In thin films the theoretical situation is considerably simpler because in certain experimental circumstances which can easily be achieved, it is necessary only to solve the one-dimensional heat flow equation ( $\$ 4$ ). Thus quantitative comparisons of experiment with models can be expected, and insights into the more complicated three-dimensional geometry can hopefully be achieved. Such comparisons are shown in $\S 4$ and the implications for $J_{c}$ stability are discussed in $\S 6$.

\subsection{Thermal propagation}

The propagation velocity of a NS boundary can be positive, negative or zero depending on the power dissipated per unit area in the normal region and the length of the normal region $L_{\mathrm{n}}$. For zero velocity, the static heat flow equations can be solved analytically to obtain values of $\left[\rho_{\mathrm{n}} J^{2} d\right]_{0}$ as a function of $L_{\mathrm{n}}$. Here $\rho_{\mathrm{n}}$ is the normal state resistivity and $d$ the film thickness. This solution separates the conditions of positive velocities $\rho_{\mathrm{n}} J^{2} d>\left[\rho_{\mathrm{n}} J^{2} d\right]_{0}$ and negative velocities $\rho_{\mathrm{n}} J^{2} d<\left[\rho_{\mathrm{n}} J^{2} d\right]_{0}$.

The one-dimensional static heat flow equation for superconducting films has also been analysed by Skocpol et al (1974) to explain hot spots in very small microbridges. However, the boundary conditions appropriate to the microbridge $\left(T=T_{\mathrm{b}}\right.$ at the ends of the microbridge) lead to results significantly different from those used in $\S 4$, which are appropriate to long films which cover most of the substrate area.

In the limit of an infinitely long normal region (hot spot), BR have shown an analytical expression relating the propagation velocity $v$ to $\rho_{\mathrm{n}} J^{2} d$. In $\S 2$ excellent quantitative agreement with this model is shown. However, this solution is invalid when $L_{\mathrm{n}}$ is less than the dynamic thermal healing length $\eta^{*}$. In $\S 3$, a new model is presented which makes use of a simple energy balance and seems to give reasonable quantitative predictions for the measured $L_{\mathrm{n}}$ dependence of $v$ when $L_{\mathrm{n}} \ll \eta^{*}$.

For propagation in sapphire substrates, $\eta^{*}$ can be of the order of $1 \mathrm{~cm}$. When $L_{\mathrm{n}}$ is much smaller than this, however, heat transfer from the film to sapphire substrate becomes a bottleneck, and thermal propagation occurs in the film alone. This bottleneck also occurs for static solutions, and is the basis for a quantitative understanding of the experimental data presented in $\$ 4$. Similar calculations for superconducting films on thermally insulating substrates (in which case propagation is also dominated by the film alone) show clearly why sapphire substrates provide such excellent stabilisation of $J_{c}(\S 6)$.

\subsection{Switch applications}

Superconducting switches have been considered by BR for cryotrons and more recently for power applications (Gray and Fowler 1978, Ulbricht 1979, Gray et al 1979). In general, thin films offer greater potential for repetitive switches because of the far shorter time for recovery to the superconducting state (Gray and Fowler 1978). It can be shown (Gray et al 1980, Gray and Fowler 1978) that high $\rho_{\mathrm{n}}$ (which precludes normal metal stabilising) and high $J_{\mathrm{c}}$ are necessary for an efficient switch. Since the power per unit volume $\rho_{\mathrm{n}} J^{2}$ 
(greater than about $10^{9} \mathrm{~W} \mathrm{~cm}^{-3}$ ) is therefore extremely high (Gray and Fowler 1978), the device must be quickly (about $\mu \mathrm{s}$ ) driven normal and the current commutated into the load to avoid fusing at the initial hot spot.

One solution is to uniformly switch the entire superconductor by an external perturbation (e.g. heat pulse), although this can be difficult and inefficient (i.e. expensive). Thermal propagation from a limited number of externally generated hot spots offers the potential to avoid these difficulties, at least for applications such as a superconducting fault current limiter (SCFCL), in which the switching time (greater than about $100 \mu \mathrm{s}$ ) is long enough (Gray and Fowler 1978).

Thermal propagation can also provide protection of superconducting switch elements against fusing from accidental hot spots. The results presented in $\S 5$ can be used to calculate the effects of such an accidental breakdown.

\subsection{Experimental details}

Thermal propagation measurements were conducted in liquid helium at $4.2 \mathrm{~K}$. Specially made, extremely low inductance strip lines were used throughout the circuit containing the superconducting element as well as the room-temperature shunt and current measuring resistors. The calculated inductance was considerably less than the upper limit of the measured inductance of $0.2 \mu \mathrm{H}$. These cryogenic feedthroughs and the slow current pulse supplying the sample were rated at $500 \mathrm{~A}$, while typical critical currents were 100-200 A. Niobium-tin superconducting composite strips were ultrasonically soldered with indium to the ends of the films for the current. The measured contact resistance was less than $0.2 \mu \Omega$ at $170 \mathrm{~A}$, leading to a negligible dissipation of $6 \mathrm{~mW}$.

Voltage probes were attached to the films with indium and considerable care was taken to keep the mutual inductance to a minimum. The same care was taken with the roomtemperature resistor used to measure the current in the superconductor.

Switching was initiated by various external perturbations as well as the self-current. These included pulses of light, magnetic field and heat from a normal metal film (deposited on the reverse side of sapphire substrates only).

Wide-band amplifiers were used and the results recorded on film from oscilloscope traces. The repetition rate was adjusted so that successive switching events were independent.

Niobium nitride samples were prepared (Kampwirth and Gray 1981) by a reactive sputtering process using an S-gun magnetron sputtering system with a niobium target and an argon sputtering gas doped with 10-20\% nitrogen. Substrates were allowed to reach ambient temperature (about $200^{\circ} \mathrm{C}$ ) or heated independently to temperatures up to $500^{\circ} \mathrm{C}$. For pure niobium samples the nitrogen gas is eliminated.

The target for $\mathrm{Nb}_{3} \mathrm{Sn}$ samples is made (Kampwirth et al 1977) of compacted powders of niobium and tin which were partially reacted at $750^{\circ} \mathrm{C}$. Sapphire substrates were heated to about $800^{\circ} \mathrm{C}$ during deposition.

Samples of $\mathrm{Nb}_{3} \mathrm{Ge}$ were also prepared on heated sapphire substrates (about $800^{\circ} \mathrm{C}$ ) using the same S-gun system but employing a germanium target with the appropriate amount of niobium pieces 'tiled' onto the surface (Kampwirth 1979).

The films were deposited so as to cover all but two narrow edges of one face of the sapphire or glass substrates. In a few cases smaller patterns were made by photoetching. Sapphire substrates were typically $5.1 \mathrm{~cm}$ long, $1.26 \mathrm{~cm}$ wide and $0.05 \mathrm{~cm}$ thick. Some samples used substrates cut to smaller lengths or widths. 


\section{Broom and Rhoderick model for thermal propagation $\left(L_{n} \gg \eta^{*}\right)$}

Thermal propagation in superconducting films in the limit of an infinitely long normal zone has been discussed previously in the literature. BR determined an analytical solution to the time-dependent heat flow equation which, however, required several assumptions.

Firstly, the sizes of the normal and superconducting regions were assumed to be infinite and the current density $J$ was assumed to be constant, so that the shape of the temperature profile (figure 1) remains invariant and moves at constant velocity.

Secondly, it was assumed that the heat trasnfer $Q$ to the helium bath (at temperature $T_{\mathrm{b}}$ ) can be approximated by

$$
\dot{Q}=F \Delta T=F\left(T-T_{\mathrm{b}}\right)
$$

and that $F$, the specific heat per unit volume $C_{\mathrm{v}}$ and thermal conductivity $K$ are temperature independent. These assumptions are strictly valid only in the limit $\Delta T \ll T$. Unfortunately, in most situations of practical interest, $\Delta T \geqslant T$, in which case $Q$ may not be proportional to $\Delta T$ and $C_{v}$ and $K$ vary between linear and cubic dependences on $T$, according to whether conduction electrons or phonons dominate, respectively. Although the use of these approximations is therefore questionable, our experiments are in good agreement with the calculations if $F, C_{\mathrm{v}}$ and $K$ are evaluated at $T_{\mathrm{c}}$, the superconducting transition temperature. This choice seems the most reasonable since it is the velocity of the NS boundary (at $T=T_{\mathrm{c}}$ ) which is of interest, but it implies implicitly that the detailed behaviour away from the Ns boundary is unimportant to the propagation velocity.

\subsection{Thermally insulating substrate}

For a film of thickness $d$ on a thermally insulating substrate, the formal BR solution for the propagation velocity $v$ is

$$
v=\frac{\alpha-1}{\alpha^{1 / 2}} \frac{1}{C_{\mathrm{v}}^{\prime}}\left(\frac{F K^{\prime}}{d}\right)^{1 / 2}
$$

where throughout this paper $C_{\mathrm{v}}^{\prime}$ and $K^{\prime}$ refer to thermal properties of a superconducting film while $C_{\mathrm{v}}$ and $K$ refer to sapphire substrates. In equation (1),

$$
\alpha \equiv\left(T_{\infty}-T_{\mathrm{c}}\right) /\left(T_{\mathrm{c}}-T_{\mathrm{b}}\right)
$$

where $T_{\infty}$ is the limiting film temperature far into the normal region. Thus $T_{\infty}-T_{\mathrm{b}}=$ $\rho_{\mathrm{n}} J^{2} d / F$, where $\rho_{\mathrm{n}}$ is the normal state resistivity of the superconducting film. Note that $\rho_{\mathrm{n}} J^{2} d$ is the power dissipated per unit area in the normal region of the film. It is convenient to define $W_{0} \equiv F\left(T_{\mathrm{c}}-T_{\mathrm{b}}\right)$ to be the power unit area of the helium-solid boundary for

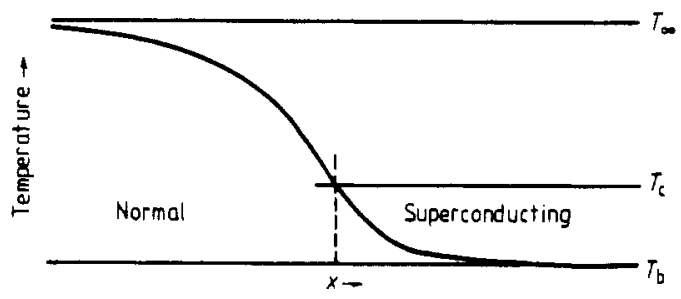

Figure 1. Schematic picture of the temperature profile at a normal-superconductor (NS) boundary. 
a temperature discontinuity of $T_{\mathrm{c}}-T_{\mathrm{b}}$. Steward (1978) has measured the steady state value of $W_{0}$ to be weakly temperature dependent, and approximately $1 \mathrm{~W} \mathrm{~cm}^{-2}$ for $T_{\mathrm{c}}-T_{\mathrm{b}} \simeq 10 \mathrm{~K}$. Note that here and throughout this paper, the effect of different solid surfaces on $F$ is ignored.

Using the above relationships it is easy to show that

$$
\alpha=\left(\rho_{\mathrm{n}} J^{2} d / W_{0}\right)-1
$$

and the zero velocity condition from equation (1) is $\alpha=1$ or $\rho_{\mathrm{n}} J^{2} d=2 W_{0}$. The factor of two accounts for the heat necessarily conducted into the superconducting regions and the fact that $T_{\infty}>T_{\mathrm{c}}$. For $\rho_{\mathrm{n}} J^{2} d<2 W_{0}$, the velocity is negative and the normal region collapses.

Equation (1) can be rewritten as

$$
\varphi \equiv \frac{\alpha-1}{\alpha^{1 / 2}}=v C_{v}^{\prime}\left(\frac{d}{F K^{\prime}}\right)^{1 / 2}
$$

and solved for $\alpha$ and hence $\rho_{\mathrm{n}} J^{2} d / W_{0}$. The result is

$$
\frac{\rho_{\mathrm{n}} J^{2} d}{W_{0}}=1+\frac{1}{4}\left[\varphi+\left(\varphi^{2}+4\right)^{1 / 2}\right]^{2}
$$

which is readily used to compare with experimental measurements. It can be easily shown that for $\varphi \gg 1$ (i.e. large velocity) the solution is independent of $W_{0}$ :

$$
\rho_{\mathrm{n}} J^{2} d \simeq W_{0} \varphi^{2}=v^{2} C_{\mathrm{v}}^{\prime 2} d\left(T_{\mathrm{c}}-T_{\mathrm{b}}\right) / K^{\prime} .
$$

Our experiments to test this model used $1 \mu \mathrm{m}$ thick niobium films on glass substrates. Handbook values (Touloukian 1970) of $C_{v}^{\prime}$ and $K^{\prime}$ are used, except that the Wiedermann-Franz law tells us to reduce the electronic contribution to $K^{\prime}$ by the ratio of the electrical conductivities: the handbook value is about $10^{7} \mathrm{~S} \mathrm{~cm}^{-1}$, while in our films it is about $3.3 \times 10^{5} \mathrm{~S} \mathrm{~cm}^{-1}$. This leads to a factor of about 30 , so that $K_{\mathrm{el}}^{\prime}\left(T_{\mathrm{c}}\right) \simeq 8 \times 10^{-3} \mathrm{~W} \mathrm{~cm}^{-1} \mathrm{~K}^{-1}$. The phonon contribution to $K^{\prime}$ is difficult to evaluate and, although its contribution is negligible in pure metals, it may be appreciable compared with the reduced electronic conductivity in our impure films. Neglecting $K_{\mathrm{ph}}^{\prime}$ and using $C_{\mathrm{v}}^{\prime}\left(T_{\mathrm{c}}\right) \simeq 0.015 \mathrm{~J} \mathrm{~cm}^{-3} \mathrm{~K}^{-1}$ and $W_{0}=1 \mathrm{~W} \mathrm{~cm}^{-2}$ results in the full curve of figure 2.

Experimental velocities are obtained by measuring the current and voltage in a superconducting film as a function of time after forming a hot spot by a localised heat pulse (figure 3). Their ratio is the resistance $R$, so that, for a film of width $w, L_{\mathrm{n}}=R d w / \rho_{\mathrm{n}}$ and $v=\frac{1}{2} \mathrm{~d} L_{\mathrm{n}} / \mathrm{d} t$ (the factor of two occurs because propagation is in both directions away from the initial hot spot). The data taken on two different samples are shown (figure 2) to be in good agreement with the calculation using no adjustable parameters. A better fit for high velocities is possible by treating $K_{\text {ph }}^{\prime}$ as an adjustable parameter. The broken curve is for $K_{\mathrm{ph}}^{\prime}=\frac{1}{2} K_{\mathrm{el}}^{\prime}$, a reasonable value for $K_{\mathrm{ph}}^{\prime}$.

The excellent agreement with the BR model is somewhat surprising since the strongly temperature-dependent thermal properties have been approximated by their values at $T_{c}$. For example, the propagation velocity may vary for different points in the temperature profile (figure 1). At the very least the profile may be altered, making some results of the simple BR model invalid. The excellent agreement may be taken as an indication that the behaviour a distance away from the NS boundary is unimportant for the thermal propagation velocity. However, it would be difficult to confirm this unambiguously with the measurements reported in this paper. 


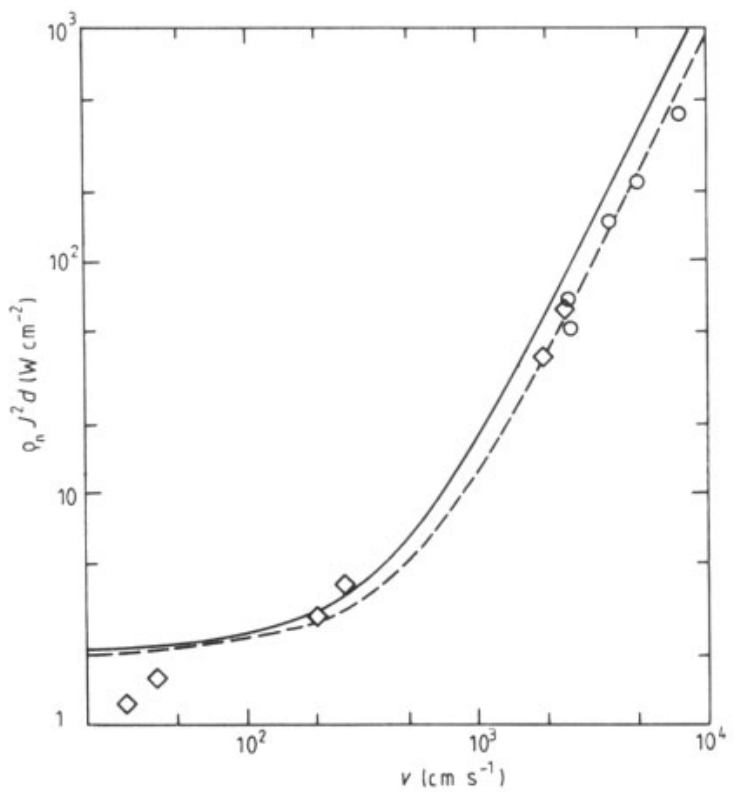

Figure 2. Propagation velocity data for two niobium films deposited on glass substrates: $\bigcirc \mathrm{Nb} 8107 ; \bigcirc \mathrm{Nb} 8103$. The full curve is the BR calculation using no adjustable parameters and only the electronic thermal conductivity of niobium. The broken curve corresponds to a phonon contribution to the thermal conductivity which is half the electron contribution.

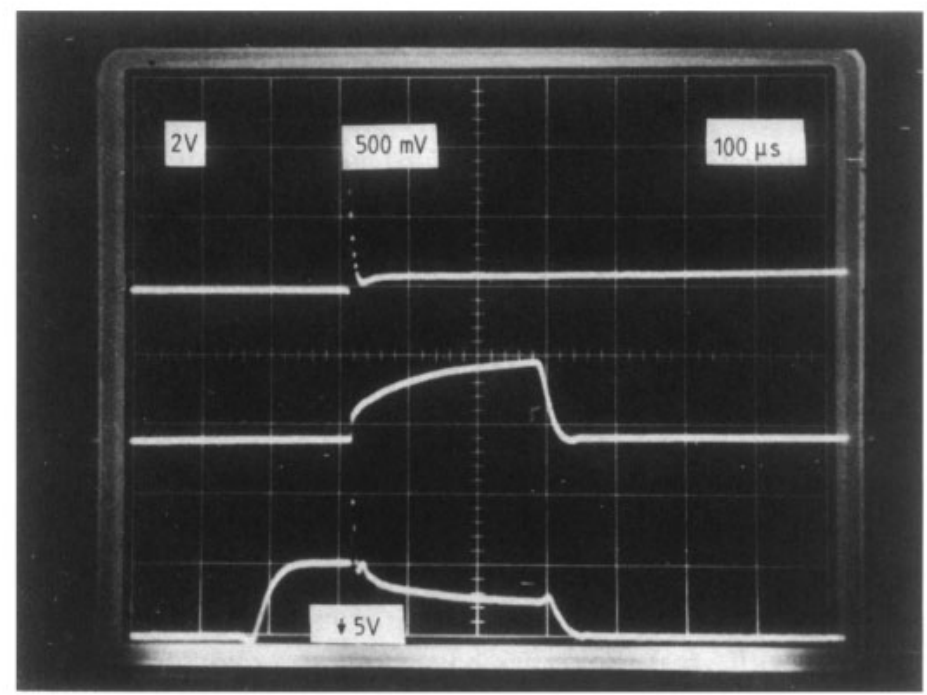

Figure 3. Oscilloscope traces of thermal propagation in a niobium film ( $\mathrm{Nb} \mathrm{8103)}$ on a glass substrate. Switching was initiated by a short light pulse (top trace) after the current in the superconductor (bottom trace) reached a steady value. Thermal propagation is seen as a steady increase in the voltage (middle trace) as the current decreases due to commutation into the shunt resistor. Finally, the current is switched off and the system recovers for the next switching event. 


\subsection{Thermally conducting substrate}

A somewhat different situation occurs for superconducting films on substrates of high thermal conductivity such as sapphire. For certain values of the parameters (cf $\S 4$ ) it is possible to neglect the temperature difference between the film and substrate, and if the thermal conductivity of the substrate is significantly better than that of the film, propagation will occur in the substrate (Newhouse 1964). The film will act as a source of heat (from its normal regions) but its temperature will follow the substrate. If the film is as wide as the substrate, the one-dimensional calculation of $\mathrm{BR}$ is valid and equation (1) becomes

$$
v=\frac{\alpha-1}{\alpha^{1 / 2}} \frac{1}{C_{\mathrm{v}}}\left(\frac{2 F K}{t}\right)^{1 / 2}
$$

where $\alpha$ is now equal to $\left(\rho_{n} J^{2} d / 2 W_{0}\right)-1$, instead of equation (2). Here $t$ is the substrate thickness and one uses $2 W_{0}$ and $2 F$ because heat can radiate into the helium from both the film and the back face of the substrate. Of course this implies no temperature gradient across the substrate-a good approximation for single crystal sapphire in which the phonon free path is limited only by the substrate thickness. In principle $C_{\mathrm{v}}$ and $K$ are evaluated for the combination of film and substrate. However, if the superconducting film is dirty and has a thickness $d$ much less than $t$, the contributions of the film to $K$ and $C_{\mathrm{v}}$ can be negligible. For the experiments reported here the film contributes about $1 \%$ to $C_{v}$ and even less to $K$. In such a case, $K$ can be evaluated from the one-dimensional diffusion equation for phonons (Kittel 1968) in the substrate, $K \simeq C_{\mathrm{v}} s l_{\mathrm{ph}}$ where $s$ is the phonon velocity and $l_{\text {ph }}$ the phonon mean free path. In the case of a single crystal substrate it is reasonable to set $l_{\mathrm{ph}}$ equal to $t$. Equation (6) can then be written as

$$
\frac{v}{s}=\frac{\alpha-1}{\alpha^{1 / 2}}\left(\frac{2 F}{s C_{v}}\right)^{1 / 2}
$$

where $C_{\mathrm{v}}$ stands for $C_{\mathrm{v}}\left(T=T_{\mathrm{c}}\right)$. The velocity has been normalised to $s$ since physically it can never be greater than $s$, the speed of ballistic heat propagation. The value of $s$ $\left(7.5 \times 10^{5} \mathrm{~cm} \mathrm{~s}^{-1}\right)$ is chosen as a suitable average of the transverse $\left(6.45 \times 10^{5} \mathrm{~cm} \mathrm{~s}^{-1}\right)$ and longitudinal $\left(10.9 \times 10^{5} \mathrm{~cm} \mathrm{~s}^{-1}\right)$ modes (Kaplan 1979), realising that at low temperatures the density of transverse states is eight times larger. Faster propagation is presumably possible if sufficient energy is available to make the faster, but fewer, longitudinal models effective. Note that propagation in the film alone (e.g. niobium on glass substrates) is not limited to the sound velocity since the conduction electrons can conduct heat at the Fermi velocity (Gray and Fowler 1978). In practice, the limited electron mean free path prevents this.

Because heat radiates from both surfaces of the substrate, $T_{\infty}-T_{\mathrm{b}}=\rho_{\mathrm{n}} J^{2} d / 2 F$ and

$$
\alpha=\left(\rho_{\mathrm{n}} J^{2} d / 2 W_{0}\right)-1 .
$$

Therefore the zero velocity condition $(\alpha=1)$ yields $\rho_{\mathrm{n}} J^{2} d=4 W_{0}$ for sapphire substrates.

The solution of this equation for finite velocity is shown as a full curve in figure 4 , along with experimental results for a $\mathrm{Nb}_{3} \mathrm{Sn}$ film on a $0.5 \mathrm{~mm}$ thick sapphire substrate. The various symbols represent different values of the length of the normal region $L_{\mathrm{n}}$. There is a clear dependence of $v$ on length which has not been previously reported in the literature and was not found for propagation on glass substrates. The data are in best agreement using $n o$ adjustable parameters with the BR calculation $\left(L_{n} \rightarrow \infty\right)$ for the greatest length. 


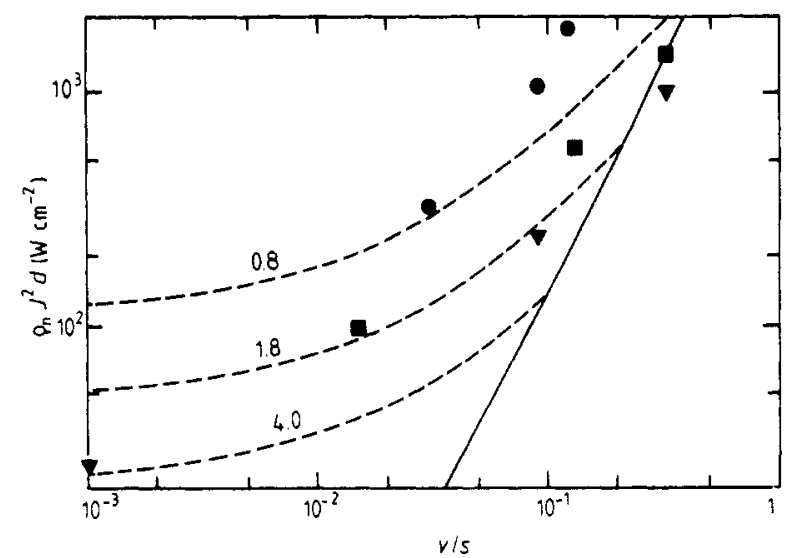

Figure 4. Thermal propagation data for $\mathrm{a} \mathrm{Nb}_{3} \mathrm{Sn}$ sample (NS 8008) on a sapphire substrate. The symbols correspond to different hot spot lengths, $L_{\mathrm{n}}: 0.8 \mathrm{~mm} ; \quad \square \mathrm{mm}$; $\nabla 4.0 \mathrm{~mm}$. The full curve is the prediction of the $\mathrm{BR}$ model for propagation in the substrate with no adjustable parameters. The broken curves are the predictions of the energy balance model for the values of $L_{n}$ indicated. The velocity of sound in sapphire, $s$, was chosen to be $7.5 \times 10^{5} \mathrm{~cm} \mathrm{~s}^{-1}$ (see text).

\subsection{Conclusion}

In summary, the BR model is quantitatively confirmed for thermal propagation in the film or substrate if the thermal properties are evaluated at $T_{\mathrm{c}}$. This conclusion suggests that only the conditions occurring right at the NS boundary are important for the determination of the propagation velocity. The next section presents a model for understanding the new dependence of $v$ on $L_{\mathrm{n}}$ found experimentally for sapphire substrates on which $L_{\mathrm{n}}<\eta^{*}$.

\section{Energy balance model of thermal propagation $\left(L_{\mathrm{n}} \ll \eta^{*}\right)$}

One can get a feeling for the different thermal propagation behaviour (i.e. length dependence) of glass and sapphire substrates by comparing the characteristic length scales $\eta$ of the time-independent heat flow equation:

$$
\eta=(K t / 2 F)^{1 / 2}
$$

is called the static thermal healing length. Note that the factor of two in $\eta$ is for the thermally conducting sapphire substrates since heat flows from both surfaces to the helium. It is absent for films on thermally insulating (glass) substrates.

Evaluating $\eta$ for glass and sapphire substrates gives an indication as to why the propagation velocities behave differently. For $\mathrm{Nb}_{3} \mathrm{Sn}$ with $T_{\mathrm{c}}=17 \mathrm{~K}$, the thermal conductivity is $0.02 \mathrm{~W} \mathrm{~cm}^{-1} \mathrm{~K}^{-1}$ (Cody and Cohen 1964), and the much thicker sapphire substrate dominates. Thus, $\eta=(K t / 2 F)^{1 / 2}=4.2 \mathrm{~cm}$. On the other hand, for our $\mathrm{Nb}$ film $\left(T_{\mathrm{c}}=9.2 \mathrm{~K}\right)$ on a glass substrate, $K^{\prime}=0.012 \mathrm{~W} \mathrm{~cm}^{-1} \mathrm{~K}^{-1}$ (see $\S 2$ ) and $\eta_{\mathrm{f}}=\left(K^{\prime} d / F\right)^{1 / 2} \simeq 25 \mu \mathrm{m}$. For the propagation studies in niobium films on glass substrates reported in the previous section, the initial hot spot was $2 \mathrm{~mm}$ long so that $L_{\mathrm{n}} \gg 2 \eta_{\mathrm{f}}$ and it is reasonable that the BR model should and does apply (see figure 2). For the sapphire substrates, $L_{\mathrm{n}}<\eta$, so a length dependence is not surprising (as shown in figure 4). 


\subsection{Energy balance model}

To quantify the length dependence of thermal propagation at finite velocities, it is necessary to go beyond the BR model and consider the energy balance in the film-substrate combination. (This model can be generalised to a film on a thermally insulating substrate in an obvious way.) The total heat energy $\delta Q_{0}$ dissipated in a time $\delta \tau$ is given by

$$
\delta Q_{0}=\left(\rho_{\mathrm{n}} J^{2} d-\left[\rho_{\mathrm{n}} J^{2} d\right]_{0}\right) w L_{\mathrm{n}}(\tau) \delta \tau
$$

where $\left[\rho_{\mathrm{n}} J^{2} d\right]_{0}$ is the solution of the steady state equation given in the next section, and approximately accounts for the heat losses to the liquid helium. It is approximate because the steady state solution is only valid for $\tau \gg \tau_{d} \sim C_{v} \eta^{2} / K$, the diffusion time, and this may not be valid in dynamic situations. Propagation at a velocity $v$ requires a minimum energy to supply the enthalpy difference $\delta \mathscr{H}$ to the normal state. Thus

$$
\delta \mathscr{H}=2 w t v \delta \tau \int_{T_{\mathrm{b}}}^{T_{\mathrm{c}}} C_{\mathrm{v}}(T) \mathrm{d} T
$$

where the factor of two is because propagation is in both directions and the integral is the specific enthalpy difference per unit volume $\Delta H$ (which equals $\Varangle C_{\mathrm{v}} T_{\mathrm{c}}$ since $C_{\mathrm{v}}(T) \propto T^{3}$ and $T_{\mathrm{c}} \gg T_{\mathrm{b}}$ ). Now $\delta Q_{0}$ is certainly greater than $\delta \mathscr{H}$ because some energy travels further into the still superconducting region and some is consumed in heating the normal region to $T>T_{\mathrm{c}}$. Therefore, setting them equal provides a lower limit on $\rho_{\mathrm{n}} J^{2} d$ given by

$$
\rho_{\mathrm{n}} J^{2} d \geqslant\left[2 t \Delta H v+4 W_{0} \eta \tanh (L / 2 \eta)\right] / L_{\mathrm{n}} .
$$

The results of evaluating equation (12) for various values of $L_{\mathrm{n}}$ corresponding to the experimental data of figure 4 are shown as broken curves in this figure. The agreement is surprisingly good for low velocities and seems to indicate that the inequality is satisfied at higher velocity.

The maximum velocity measured in these experiments is $0.37 s$, where $s$ is the sound velocity in sapphire. It is unlikely that values above about $0.5 s$ will be attainable because most of the phonon velocities are not exactly in the propagation direction along the substrate.

Equation (12) for the energy balance model will intersect equation (6) of the BR model at a value of $L_{\mathrm{n}}$ which can be associated with the dynamic thermal healing length $\eta^{*}$ :

$$
\eta^{*}=\frac{t v \Delta H / W_{0}+2 \eta}{1+\frac{1}{4}\left[\varphi+\left(\varphi^{2}+4\right)^{1 / 2}\right]^{2}}
$$

where $\varphi=\eta v / s t$ for propagation in a sapphire substrate. The crossover between these behaviours must be smooth, as in the steady state solutions of $\S 4$; however, the intersection gives a specific definition to $\eta^{*}$ as in the case of the static thermal healing length $\eta$. Unfortunately, the extrapolation of the BR model (equation (6)) to such small values of $L_{\mathrm{n}}$ is not obviously valid especially at high velocities. There are different thermal healing lengths in the BR model for the normal and superconducting regions:

$$
\eta_{\mathrm{s}, \mathrm{n}}=\frac{\eta / \varphi}{\left[1+(2 / \varphi)^{2}\right]^{1 / 2} \pm 1}
$$

For very large $v$ (and hence $\varphi$ ), $\eta_{\mathrm{B}}$ is very short and $\eta_{\mathrm{n}}$ very long. The BR model is strictly valid only if $L_{\mathrm{n}} \gg \eta_{\mathrm{n}}$, leaving a large gap in $L_{\mathrm{n}}$ values, between which the BR and energy balance models are valid. To overcome this difficulty, we speculate that the BR model can 
be extended to values of $L_{\mathrm{n}}$ as small as $\eta^{*}$, relying for justification on the apparent unimportance of regions far away from the NS boundary to thermal propagation (as evidenced by the excellent agreement with experiment using the thermal properties at $T_{\mathrm{c}}$ shown in figures 2 and 4 ).

\subsection{Narrow films}

Some experiments were performed using narrow $(2 \mathrm{~mm}$ ) niobium films on a $1 \mathrm{~cm}$ wide sapphire substrate. The measurements (figure 5) made with constant current density show the slope changing when the Ns boundary arrives at one end of the film before the other (Newhouse 1964). It required about ten times the calculated power (equations (6) and (12)) for the measured average propagation velocity. Naive considerations would indicate that this should equal the ratio of the substrate to film width, i.e. about five.

\subsection{Conclusions}

In summary, because the thermal healing length is so long for sapphire substrates, the BR model for thermal propagation is only approximately confirmed. However, a new energy balance model is apparently capable of quantitatively explaining the new, unexpected length dependence of the propagation velocity for $L_{\mathrm{n}} \ll \eta^{*}$.

\section{Steady state solutions}

There is also a significant difference in the steady state (zero propagation velocity) solutions when using glass and sapphire substrates. To have a basis for discussion the steady state heat flow equations are reviewed first.

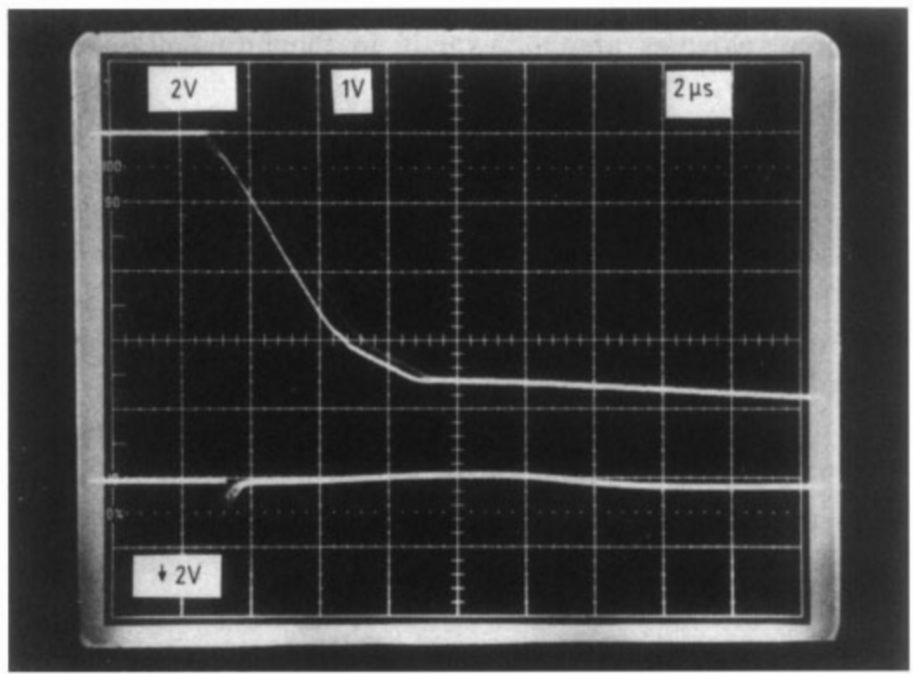

Figure 5. Oscilloscope traces of thermal propagation in a narrow ( $2 \mathrm{~mm}$ wide) niobium film (Nb 8105) on a $1 \mathrm{~cm}$ wide sapphire substrate. Switching was initiated by a $2 \mathrm{~mm}$ long light pulse approximately in the middle of the $15 \mathrm{~mm}$ long film. The breaks in the slope of the voltage (upper trace) correspond to the Ns boundary reaching the ends of the film. The lower trace shows the current to be constant during the propagation. 


\subsection{Steady state heat flow equations}

Consider a superconducting film in perfect thermal contact with, and on one side of, a high thermal conductivity substrate such as sapphire. If the film is as wide as the substrate, the following (Skocpol et al 1974) one-dimensional steady state heat flow equations are valid: in the normal zone

$$
-\left(\partial^{2} T_{\mathrm{n}} / \partial x^{2}\right)+\left(T_{\mathrm{n}}-T_{\mathrm{b}}\right) / \eta^{2}=\rho_{\mathrm{n}} J^{2} d / K t
$$

and in the superconducting zones

$$
-\left(\partial^{2} T_{\mathrm{s}} / \partial x^{2}\right)+\left(T_{\mathrm{s}}-T_{\mathrm{b}}\right) / \eta^{2}=0
$$

where $\eta$ is the static thermal healing length (equation (9)). The general solutions are

$$
T_{\mathrm{n}, \mathrm{s}}=A_{\mathrm{n}, \mathrm{s}} \cosh (x / \eta)+B_{\mathrm{n}, \mathrm{s}} \sinh (x / \eta)+C_{\mathrm{n}, \mathrm{s}} .
$$

Define the middle of the normal zone as $x=0$, and assume it is in the middle of a substrate of length $L$. There are two sets of boundary conditions: at the NS boundary ( $x= \pm \frac{1}{2} L_{\mathfrak{n}}$ ), the temperature equals $T_{\mathfrak{c}}$, and its derivative (hence heat flow) must be continuous; through the end of the substrate $\left(x= \pm \frac{1}{2} L\right)$ there is negligible heat flow, so we set $\mathrm{d} T_{\mathrm{s}} / \mathrm{d} x=0$. The resulting solution is

$$
\frac{\rho_{\mathrm{n}} J^{2} d}{2 W_{0}}=\frac{\operatorname{cotanh}\left(L_{\mathrm{n}} / 2 \eta\right)-\tanh \left(L_{\mathrm{n}} / 2 \eta\right)}{\operatorname{cotanh}(L / 2 \eta)-\tanh \left(L_{\mathrm{n}} / 2 \eta\right)} .
$$

In the limit $L / 2 \eta \rightarrow \infty$, this gives

$$
\rho_{\mathrm{n}} J^{2} d=2 W_{0}\left(1+\operatorname{cotanh}\left(L_{\mathrm{n}} / 2 \eta\right)\right)
$$

and if $L_{\mathrm{n}} / 2 \eta \rightarrow \infty$ also, this yields $\rho_{\mathrm{n}} J^{2} d=4 W_{0}$ which is exactly the BR result for zero velocity. For $L_{\mathrm{n}} / 2 \eta \ll 1$, however, the solution of equation (17) reduces to

$$
\rho_{\mathrm{n}} J^{2} d / 2 W_{0}=\left(2 \eta / L_{\mathrm{n}}\right) \tanh (L / 2 \eta) \text {. }
$$

Thus at $L_{\mathrm{n}}^{*}=\eta \tanh (L / 2 \eta)$ there is a crossover from $\rho_{\mathrm{n}} J^{2} d=4 W_{0}(\eta \tanh (L / 2 \eta)) / L_{\mathrm{n}}$ to $\rho_{\mathrm{n}} J^{2} d=4 W_{0}$. For an infinite substrate $(L \gg 2 \eta)$ the crossover is at $L_{\mathrm{n}}^{*}=\eta$, the static thermal healing length.

\subsection{Stable hot spot}

These have been studied previously in very thin aluminium films (Gray 1976) on glass substrates. One important difference was the smaller values of $T_{\text {cAl }} \sim 1.59 \mathrm{~K}$ and $T_{\mathrm{b}} \simeq 1.15 \mathrm{~K}$ which resulted in $W_{0} \simeq 0.03 \mathrm{~W} \mathrm{~cm}^{-2}$.

The present experimental studies of stable hot spots generated new and surprising results when compared with steady state solutions of the heat flow equations. For thermally conducting substrates there are two distinct regimes with different thermal healing lengths. At low power and large $L_{n}$, the film and substrate are well coupled (temperatures approximately equal) and obey equation (17) with $\eta=(K t / 2 F)^{1 / 2}$. On the other hand, for high power and short $L_{\mathrm{n}}$ the film and substrate temperatures are not necessarily equal and the substrate replaces the helium as the most important weakly coupled low-temperature thermal reservoir. Thus the relevant thermal healing length becomes $\eta_{\mathrm{f}}=\left(K^{\prime} d / F^{\prime}\right)^{1 / 2} \ll \eta$ where $F^{\prime}$ refers to the film-substrate boundary. In the following subsections, experimental confirmation of these concepts is reported, and in $\S 5$, 
the decoupling of the film and substrate is generalised to the energy balance and BR models for finite propagation velocity.

\subsection{Experimental confirmation}

The experimental thermal propagation studies were normally conducted with a shunt resistor connected across the current leads to the sample where they emerged from the cryostat. When no shunt was used, the initial hot spot would propagate across the entire sample length unless the current was interrupted prematurely. With the shunt, the hot spot could propagate to a finite size, less than the full length of the sample, and then stop. The final length $L_{\mathrm{n}}$ depends on the shunt resistor and the initial current, and is the intersection of the electrical load line with the steady state solution (equation (17)).

The dependence of $L_{\mathrm{n}}$ on $\rho_{\mathrm{n}} J^{2} d$ for measured stable hot spots is compared with the predictions of equation (17) for two samples on $5.1 \mathrm{~cm}$ long sapphire substrates in figure 6 . Note that the voltage probes were $3 \mathrm{~cm}$ apart for the $\mathrm{Nb}_{3} \mathrm{Sn}$ sample, so that $L_{\mathrm{n}}$ is limited to that value. Points plotted at $3 \mathrm{~cm}$ can have arbitrarily large $\rho_{\mathrm{n}} J^{2} d$ and would have intersected the steady state solution at $L_{\mathrm{n}}>3 \mathrm{~cm}$. The full curve is equation (17) evaluated for the $\mathrm{Nb}_{3} \mathrm{Sn}$ sample with $L=5.1 \mathrm{~cm}$ and $\eta=4.2 \mathrm{~cm}$; however, the curve for the $\mathrm{NbN}$ sample $(\eta=3.2 \mathrm{~cm})$ is almost indistinguishable on this graph. Note that for $L_{\mathrm{n}}=L \neq 0$, equation (17) reduces to $\rho_{n} J^{2} d=2 W_{0}$ as shown in figure 6 . With no heat flow from the ends of a finite length normal film-substrate combination, the temperature must be uniform along the length, dissipating $W_{0}$ through each surface into the helium. The additional factor of two found in the BR model is absent because no heat flows from the ends of the normal region and $T$ is nowhere greater than $T_{\mathrm{c}}$.

For the larger values of $L_{n}$ shown in figure 6, the agreement with equation (17) is

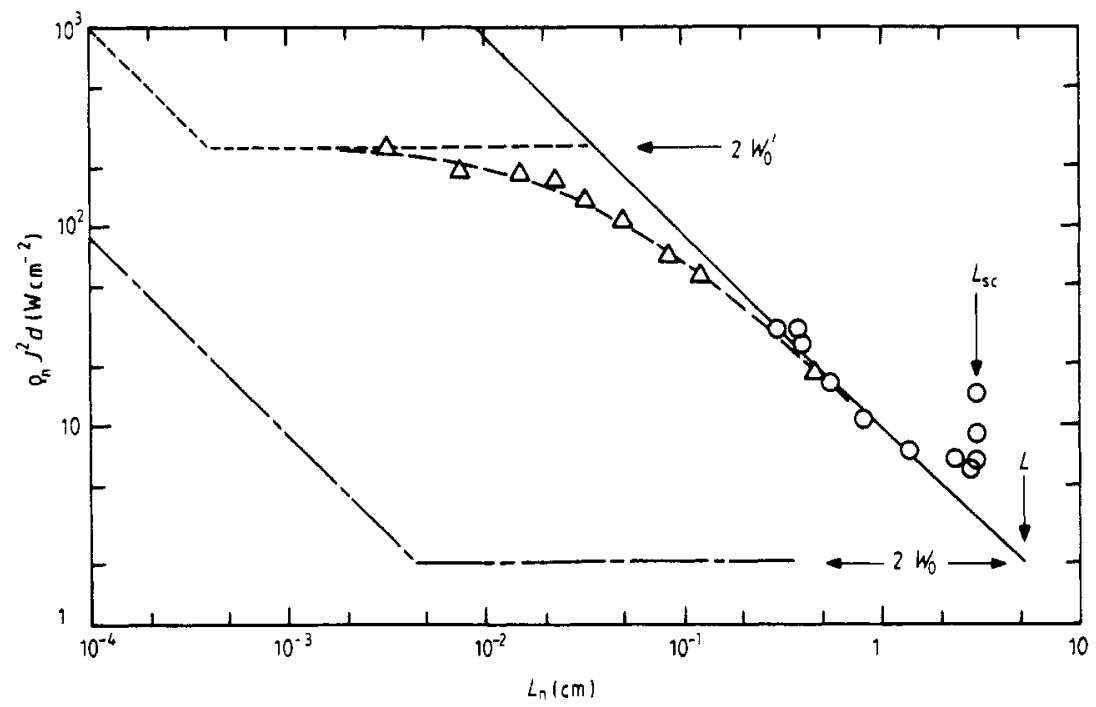

Figure 6. Stable hot spots for two films on $5.1 \mathrm{~cm}$ long sapphire substrates: $\triangle \mathrm{NbN} 8018$; $\bigcirc \mathrm{Nb}_{3} \mathrm{Sn} 8008$. The $\mathrm{Nb}_{3} \mathrm{Sn}$ film was $3 \mathrm{~cm}$ long. The full curve is the prediction of the steady state heat flow (equation (17)) for the film well coupled to the substrate. The broken line is a similar calculation for the film weakly coupled to the substrate. The broken curve is the interpolation (equation (20)) between the two, with the only adjustable parameter being $W_{0}^{\prime}$. The chain line represents the expected behaviour for a film on a thermally insulating substrate. 
excellent and again uses no adjustable parameters; however, for smaller values of $L_{n}$ there appears to be a saturation. This saturation occurs when $\rho_{\mathrm{n}} J^{2} d$ is great enough that a significant part of the temperature difference $T_{c}-T_{b}$ can be maintained across the film-substrate Kapitza boundary. As a result, a smaller temperature gradient is needed in the sapphire substrate, and $\rho_{\mathrm{n}} J^{2} d$ falls below the prediction of equation (17).

\subsection{Film weakly coupled to substrate}

For sufficiently small hot spots, the entire temperature difference is across the film-substrate boundary and the steady state solution for the isolated film is applicable. In this case $\eta_{\mathrm{f}}=\left(K^{\prime} d / F^{\prime}\right)^{1 / 2}$, where $F^{\prime}$ is the heat transfer coefficient across the film-substrate boundary and $K^{\prime}$ the thermal conductivity of the film. Following the derivation in $\S 4$, it is found that for $L_{\mathrm{n}} \gg \eta_{\mathrm{f}}$

$$
\rho_{\mathrm{n}} J^{2} d=2 F^{\prime}\left(T_{\mathrm{c}}-T_{\mathrm{b}}\right) \equiv 2 W_{0}^{\prime}
$$

the single factor of two resulting again from heat flow to the still superconducting regions and the fact that $T_{\infty}$ is greater than $T_{\mathrm{c}}$ deep into the hot spot. However, there is only one cooled surface, so the additional factor of two is missing.

For intermediate values of $L_{n}$, between the well coupled and isolated film limits, it is convenient to define $T_{\mathrm{s}}$ to be the substrate temperature beneath the hot spot; then across the Kapitza boundary

$$
\rho_{\mathrm{n}} J^{2} d=2 F^{\prime}\left(T_{\mathrm{c}}-T_{\mathrm{s}}\right)
$$

while in the substrate

$$
\rho_{\mathrm{n}} J^{2} d=\left[4 F\left(T_{\mathrm{s}}-T_{\mathrm{b}}\right) \eta / L_{\mathrm{n}}\right] \tanh (L / 2 \eta) .
$$

Eliminating $T_{\mathrm{s}}$ yields

$$
\rho_{\mathrm{n}} J^{2} d=2 W_{0}^{\prime}\left(1+\frac{W_{0}^{\prime}}{W_{0}} \frac{L_{\mathrm{n}}}{2 \eta \tanh (L / 2 \eta)}\right)^{-1} .
$$

This equation, using the one adjustable parameter $W_{0}^{\prime}=125 \mathrm{~W} \mathrm{~cm}^{-2}$, is shown as the broken curve in figure 6 and is in excellent agreement with the data.

The Kapitza boundary conductance $Y_{\mathrm{K}}$ for an indium-sapphire boundary has been previously measured (Neeper and Dillinger 1964) at $4.2 \mathrm{~K}$ to be $Y_{\mathrm{K}}=$ $0.028 T^{3} \mathrm{~W} \mathrm{~cm}^{-2} \mathrm{~K}^{-1}$. This result can be extrapolated to the $T_{\mathrm{c}}$ of $15 \mathrm{~K}$ for $\mathrm{NbN}$ using the usual $T^{4}$ law (Kaplan 1979) and would imply $W_{0}^{\prime}=350 \mathrm{~W} \mathrm{~cm}^{-2}$. This is considerably larger than our value, indicating poorer thermal contact for our granular sputtered $\mathrm{NbN}$ films. This conclusion is consistent with the fact that $\mathrm{NbN}$ films deposited at $200^{\circ} \mathrm{C}$ do not adhere to the substrate as well as most other sputtered or evaporated films.

Of course when $L_{\mathrm{n}} \ll \eta_{\mathrm{f}}$ the value of $\rho_{\mathrm{n}} J^{2} d$ for the steady state solution should increase by analogy with equation (19):

$$
\rho_{\mathrm{n}} J^{2} d=2 W_{0}^{\prime} \eta_{\mathrm{r}} / L_{\mathrm{n}}
$$

where it is assumed that $\tanh \left(L / 2 \eta_{\mathrm{f}}\right) \simeq 1$. It is difficult to predict $\eta_{\mathrm{f}}$ accurately because of the uncertainty in the thermal conductivity of $\mathrm{NbN}$. An estimate of $\eta_{\mathrm{f}}$ is obtained by using the value measured (Cody and Cohen 1964) for $\mathrm{Nb}_{3} \mathrm{Sn}$ of about $0.02 \mathrm{~W} \mathrm{~cm}^{-1} \mathrm{~K}^{-1}$, which is somewhat larger than the value used for niobium in $\S 2$. Then assuming $d=1 \mu \mathrm{m}$ and $T_{\mathrm{c}}=15 \mathrm{~K}$ (for $\mathrm{NbN}$ ), $\eta_{\mathrm{f}} \simeq 4 \mu \mathrm{m}$ which is approximately $10^{4}$ times smaller than $\eta$ for the 
sapphire substrate. Equation (21) is shown as the asymptote in the upper left corner of figure 6 .

\subsection{Critical current stability}

The behaviour of stable solutions for such small values of $L_{\mathrm{n}}$ may be helpful to our understanding of the stability of the superconducting state at high current densities. The above analysis of the steady state heat flow equation predicts the minimum value of $\rho_{\mathrm{n}} J^{2} d$ for which a normal state fluctuation (perhaps due to a thermally activated flux jump) of size $L_{\mathfrak{n}}$ will propagate. It could therefore be viewed as a relationship showing how the critical current density $J_{\mathrm{c}}$ depends on the size of a normal state fluctuation. For our best $\mathrm{NbN}$ and $\mathrm{Nb}_{3} \mathrm{Sn}, \rho_{\mathrm{n}} J_{\mathrm{c}}^{2} d \simeq 2.5 \times 10^{4} \mathrm{~W} \mathrm{~cm} \mathrm{~cm}^{-2}$, and the above interpretation indicates a critical size $L_{\mathfrak{n c}} \sim 400 \AA$.

Such an interpretation is appealing because of its predictive powers. For example, it can explain the different values of $J_{c}$ for $\mathrm{NbN}$ on glass and sapphire substrates (Kampwirth and Gray 1981). The above interpretation suggests that $J_{\mathrm{c}}$ is proportional to $\left(W_{0}^{\prime}\right)^{1 / 4}$ since

$$
\rho_{\mathrm{n}} J_{\mathrm{c}}^{2} d=\left[4 K^{\prime} W_{0}^{\prime} d\left(T_{\mathrm{c}}-T_{\mathrm{b}}\right) / L_{\mathrm{nc}}^{2}\right]^{1 / 2} .
$$

This implies a smaller $J_{\mathrm{c}}$ on glass substrates for the same value of $L_{\mathrm{nc}}$ (see the bottom curve of figure 6 appropriate to glass substrates). The predicted factor of $\left(W_{0} / W_{0}^{\prime}\right)^{1 / 4}$ is in reasonable agreement with $J_{c}$ measurements (Kampwirth and Gray 1981) on wide $\mathrm{NbN}$ films (about $1-2 \mathrm{~cm}$ ). However, using equation (22), it is difficult to understand the large variations (about a factor of three) of $J_{\mathrm{c}}$ for similar samples of the same material if $L_{\mathrm{nc}}$ is constant, since variations from sample to sample of the parameters $K^{\prime}$ and $W_{0}^{\prime}$ are not likely to be large enough. If $L_{n c}$ must vary also, the model may only predict an upper limit on critical current density, unless $L_{\mathrm{nc}}$ can be obtained independently. Stability is discussed in more detail in $\S 6$.

\subsection{Conclusions}

In summary, it has been demonstrated that the solutions of the steady state heat flow equation can accurately predict stable hot spots. For high thermal conductivity substrates like sapphire, temperature gradients in the substrate must be considered in addition to the gradients in the film and the temperature drop at the Kapitza boundary of the film. In addition, sapphire substrates can stabilise superconducting films to higher values of critical current density than can thermally insulating substrates. This is because the thermal conductivity of glass substrates is too low to be effective and the thermal contact of the film to helium is much worse than to sapphire $\left(W_{0}^{\prime}=125 \mathrm{~W} \mathrm{~cm}^{-2}\right.$ for sapphire but $W_{0}=1 \mathrm{~W} \mathrm{~cm}^{-2}$ for liquid helium).

\section{Thermal propagation on sapphire substrates}

The results of the previous three sections can be combined to give the overall picture of thermal propagation of normal zones in superconducting films on sapphire substrates. A thermally conducting sapphire substrate produces four regimes to be considered. For long hot spots, the film and substrate are well coupled thermally and either the BR model $\left(L_{\mathrm{n}}>\eta^{*}\right)$ or the energy balance model $\left(L_{\mathrm{n}}<\eta^{*}\right)$ applies. For short hot spots, the film is 
weakly coupled to the substrate which replaces the liquid helium as the low-temperature thermal reservoir. There are thus two more cases: the BR model for $L_{n}>\eta_{f}^{*}$ and the energy balance model for $L_{n}\left\langle\eta_{\mathrm{f}}^{*}\right.$. The validity and usefulness of this picture will be strikingly demonstrated by the quantitative predictions of a curious repetitive switching phenomenon described later in this section.

\subsection{Propagation velocities}

For hot spots with $L_{\mathrm{n}} \gg \eta^{*}$ the propagation velocity is given by equation (6) of the BR model. Here the length of the superconductor and substrate are assumed to be much larger than $\eta^{*}$. When $L_{\mathrm{n}} \ll \eta^{*}$ the energy balance model predicts the velocity through equation (12). However, for very short hot spots and high $\rho_{\mathrm{n}} J^{2} d$, propagation can occur in the film alone, independent of the substrate. Although there are no experiments in this case, we conjecture that the BR model (equation (1)) should apply for $L_{\mathrm{n}} \gg \eta^{*}$ and the energy balance model for $L_{\mathrm{n}} \ll \eta^{*}$. The latter requires an obvious modification of equation (12) into

$$
\rho_{\mathrm{n}} J^{2} d \geqslant\left(2 d \Delta H^{\prime} v+2 W_{0}^{\prime} \eta_{\mathrm{f}}\right) / L_{\mathrm{n}}
$$

where $\Delta H^{\prime}$ is the films' enthalpy difference between $T_{\mathrm{b}}$ and $T_{\mathrm{c}}$, and it is assumed that $L / 2 \eta_{\mathrm{f}} \gg 1$.

For various values of the propagation velocity (normalised to $s$, the sound velocity on sapphire) these equations, giving the asymptotic behaviours, are shown as the full and broken (without arrows) lines in figure 7 . In order to evaluate the velocity for given values of $\rho_{\mathrm{n}} J^{2} d$ and $L_{\mathrm{n}}$ the following procedure was adopted. If the BR velocity ( $v_{4}$ from equation (6)) for propagation in the sapphire was algebraically less. than the energy balance model for propagation in the sapphire $\left(v_{3}\right.$ from equation (12)) then the BR value is used $\left(v=v_{4}\right)$. If not, then $v_{3}$ is compared with the BR velocity for propagation in the film alone ( $v_{2}$ from equation (1)). Now if $v_{3}$ is greater than $v_{2}$, the velocity is $v_{3}$. If not, $v_{2}$ is compared with the energy balance model for propagation in the film alone ( $v_{1}$ from equation (23)). Again if $v_{2}$ is less than $v_{1}$ the velocity is $v_{2}$, otherwise it is $v_{1}$.

Note that $\rho_{\mathrm{n}} J^{2} d$ is normalised to $s C_{\mathrm{v}} T_{\mathrm{c}}$, so that the higher velocity data for propagation in the sapphire substrate are universal. At low velocities the results are specific to $W_{0}=1 \mathrm{~W} \mathrm{~cm}{ }^{-2}$ and $T_{\mathrm{c}}=17 \mathrm{~K}$ for $\mathrm{Nb}_{3} \mathrm{Sn}$ in which case $s C_{\mathrm{v}} T_{\mathrm{c}}=1.88 \times 10^{4} \mathrm{~W} \mathrm{~cm}^{-2}$ and $4 W_{0} / s C_{\mathrm{v}} T_{\mathrm{c}}=2.13 \times 10^{-4}$. At lower values of $L_{\mathrm{n}}$, where propagation is in the film alone, the BR solutions depend on the thermal properties of the film $\left(C_{\mathrm{v}}^{\prime}, K^{\prime}\right)$, its thickness $d$ and for small velocities the heat transfer to the sapphire $W_{0}^{\prime}$.

Negative velocities (broken lines without arrows) are solutions to the equations for $\rho_{\mathrm{n}} J^{2} d$ less than the steady state values (equation (18) and curve $\mathrm{S}$ ). The same procedure for selecting $v$ from equations (1), (6), (12) and (23) was adopted and, although it leads to a somewhat strange looking dependence on $\rho_{\mathrm{n}} J^{2} d$ and $L_{\mathrm{n}}$, we believe it is a reasonable projection (see discussion on repetitive switching later in this section).

In figure 7 , only the asymptotic solutions are shown for simplicity. The crossovers will be smooth and similar to the steady state solutions of equations (18) and (20) (figure 6). However, it is clear that the dynamic thermal healing length decreases with increasing values of the magnitude of the velocity (see equation (13)).

\subsection{Load line}

The broken curves with arrows in figure 7 are possible switching trajectories which depend 


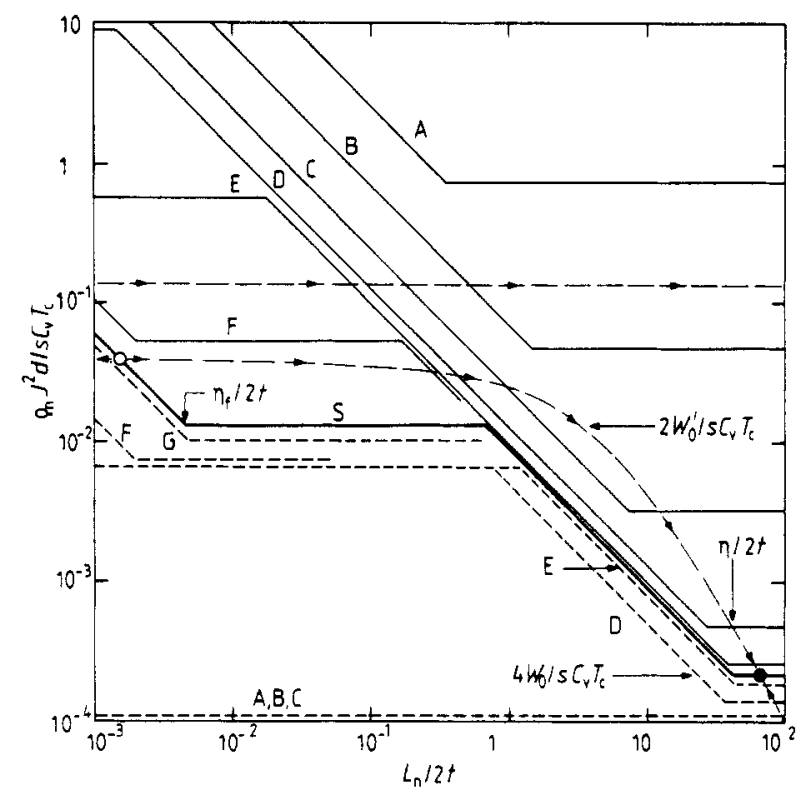

Figure 7. The full lines (broken lines without arrows) represent the value of $\rho_{n} J^{2} d$ as a function of $L_{n}$ for various positive (negative) values of velocity normalised to the velocity of sound s: A, 1; B, 0.25; C, $0.0625 ; \mathrm{D}, 0.0156 ; \mathrm{E}, 0.0039 ; \mathrm{F}, 0.00097$. The steady state solution (zero velocity) is curve $S$. The substrate length is assumed to be infinite. The broken curves with arrows are possible load line trajectories for thermal propagation. The lower trajectory represents a shunt resistor and intersects curve $\mathbf{S}$ at an unstable $(O)$ and a stable (O) equilibrium point. Values are appropriate to $\mathrm{Nb}_{3} \mathrm{Sn}$ on sapphire for which $s C_{\mathrm{v}} T_{\mathrm{c}}=1.88 \times 10^{4} \mathrm{~W} \mathrm{~cm}^{-2}, 2 t=1 \mathrm{~mm}, W_{0}=1 \mathrm{~W} \mathrm{~cm}^{-2}$ and $W_{0}^{\prime}=125 \mathrm{~W} \mathrm{~cm}^{-2}$.

on the electrical load line, i.e. the impedance and magnitude of the current source to the film. Note that curves A-S in figure 7 represent distorted current-voltage characteristics since $L_{\mathrm{n}}$ is proportional to the resistance, $V / I$, of the film and $J^{2} \propto I^{2}$. For example, the asymptotic steady state solution for $L_{\mathrm{n}} \ll \eta$ can be written as $I=4 W_{0} \eta w(\tanh (L / 2 \eta)) / V$. Note that in all cases the differential resistance is negative. The upper broken line with arrows is the load line for a perfect current source, i.e. infinite source impedance, and will be discussed later. The lower curve represents a purely resistive source impedance equal to the normal state resistance of an identical superconducting film of length 9.4t. (An example would be a shunt resistor with zero reactance.) This latter curve demonstrates the usefulness of such a diagram. The load line intersects the steady state curve, $S$, in two places. On the left the intersection is unstable because perturbations to larger $L_{\mathfrak{n}}$ yield positive velocities so that $L_{\mathrm{n}}$ grows even more, and vice versa for smaller $L_{\mathrm{n}}$. Conversely, the intersection on the right is stable. Note that one could achieve a stable solution at the intersection on the left, if a much smaller source resistance and larger initial current density were used, so that the load line intersects the steady state curve with a negative slope steeper than -1 . The relationship of the unstable equilibrium point on the left to the critical current density has been mentioned in $\S 4$, and will be discussed in greater detail in $\S 6$.

If there are reactive components to the load line impedance or in the superconducting film, the trajectory will depend on the history of the propagation. For example, a large inductance $\mathscr{L}$ will impede the commutation of current into the shunt resistor so that the effective load line is approximately a constant current source for times shorter than the 
$\mathscr{L} / R$ time. In this case of constant current, the time dependence of the propagation velocity can be determined analytically.

Clearly for a constant current, there will be a constant velocity solution of the BR equations for the given value of $\rho_{\mathrm{n}} J^{2} d$ both for propagation in the substrate, $v_{4}$ from equation (6), and in the film alone, $v_{2}$ from equation (1). These are connected by a range of $L_{\mathrm{n}}$ in which the velocity increases with $L_{\mathrm{n}}$ (and hence time) according to equation (12). In this range, it can be shown that $L_{\mathrm{n}}$ increases exponentially with time, and it is clear that the velocity, i.e. $\mathrm{d} L_{\mathrm{n}} / \mathrm{d} \tau$, also increases exponentially with time. The time to cross this range, $\tau^{\prime}$, is $\tau_{0} \ln \left(v_{4} / v_{2}\right)$. For the constant current trajectory shown in figure $7, \rho_{\mathrm{n}} J^{2} d / s C_{\mathrm{v}} T_{\mathrm{c}}=0.133$, $\tau_{0}=120 \mathrm{~ns}$ and $\tau^{\prime}=660 \mathrm{~ns}$.

\subsection{Repetitive switching}

In another example, the above considerations can be used to explain the otherwise puzzling results shown in figure 8 . These oscilloscope traces show the current in a superconducting $\mathrm{Nb}_{3} \mathrm{Sn}$ film when $5 \mathrm{~ms}$ current pulses are applied to the film and parallel shunt $R_{8}=6 \mathrm{~m} \Omega$. As the current increases from $143 \mathrm{~A}$ in $(a)$ to $150 \mathrm{~A}$ in $(b)$, breakdown begins to occur, but it is followed by recovery to the superconducting state after a delay of about $200 \mu \mathrm{s}$. At $160 \mathrm{~A}(c)$, the film breaks down to a stable steady state after recovering once or twice. However, at higher currents the recovery of superconductivity ceases completely, although there are oscillations around the steady state values (as in figure 8(c)).

Two aspects of these curves can be understood immediately. The slow recovery of current reflects the $\mathscr{L} / R_{\mathrm{s}}$ time (equal to $250 \mu \mathrm{s}$ ) where $\mathscr{L}$ is the inductance of the shunt-superconductor loop (measured to be $1.5 \mu \mathrm{H}$ in this experiment which did not use the special low inductance feedthrough). The drop in current upon breakdown is much faster, because of the shorter $\mathscr{L} /\left(R_{\mathrm{s}}+R_{\mathrm{sc}}\right)$ time, when the sample $\left(R_{\mathrm{sc}}\right)$ is normal. The normal state resistance of $440 \mathrm{~m} \Omega$ leads to a time constant of about $3 \mu \mathrm{s}$.

However, the reason for recovery and the delay time of about $200 \mu \mathrm{s}$ must also be explained. It is well known that if an element of an electrical circuit has a negative differential resistance (such as the steady state solution of equation (17) or curve $S$ of figure 7), the circuit can oscillate provided that the negative resistance balances the losses in the circuit. However, the frequency of oscillation shown in figure 8 requires an unphysically large value of circuit capacitance (about $1000 \mu \mathrm{F}$ ) to complete the tank circuit with the measured inductance. In addition, one could not understand the absence of recovery for larger currents. Therefore, the conventional negative resistance oscillator cannot explain these results.
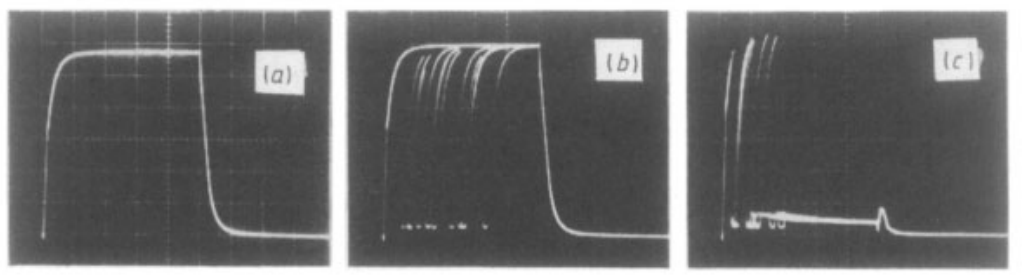

Figure 8. A series of oscilloscope traces of the current through a $\mathrm{Nb}_{3} \mathrm{Sn}$ sample (NS 7915) on a sapphire substrate showing the onset and quenching of repetitive switching as the total current increases: (a) $143 \mathrm{~A}$; (b) $150 \mathrm{~A}$; (c) $160 \mathrm{~A}$. A shunt resistor of $6 \mathrm{~m} \Omega$ is connected across the sample at room temperature and the inductance of that loop was measured as $1.5 \mu \mathrm{H}$. 
There is, however, a very good quantitative explanation of this recovery and delay time using the ideas of the previous sections embodied in figures 6 and 7. The circuit inductance is crucial since it gives the necessary delay of the current response following increases in the resistance (or $L_{n}$ ) of the superconducting film. As such the film is very quickly driven entirely normal before the current commutates significantly into the shunt. During the following period of time, the current continues to commutate towards the steady state load line (i.e. with a purely resistive shunt) represented by

$$
\rho_{\mathrm{n}} J^{2} d / \rho_{\mathrm{n}} J(0)^{2} d=\left(L_{\mathrm{s}} / L_{\mathrm{n}}\right)^{2}
$$

where $L_{\mathrm{s}}$ is the equivalent length of the shunt-about $0.048 \mathrm{~cm}$ for this experiment. Evaluating this for $L_{n}$ equal to the superconductor length of $3.5 \mathrm{~cm}$ and $I(0)=150$ A gives $\rho_{\mathrm{n}} J^{2} d \simeq 0.4 \mathrm{~W} \mathrm{~cm}^{-2}$. However, the zero velocity steady state solution (see figure 6) for $3.5 \mathrm{~cm}$ is $2.8 \mathrm{~W} \mathrm{~cm}^{-2}$, so that when $\rho_{\mathrm{n}} J^{2} d$ falls below this value, the propagation velocity becomes negative. If the hot spot then collapses faster than the current commutates back into the superconductor (i.e. the state, represented by the instantaneous values of $\rho_{\mathrm{n}} J^{2} d$ and $L_{\mathrm{n}}$, is always below the steady state solution so the velocity remains negative), the collapse will be complete and superconductivity restored. It is also necessary that, once superconducting, the critical current recovers faster than the current commutates into the superconductor to prevent premature switching back into the normal state. This final point will be discussed first, followed by a quantitative numerical calculation of $L_{n}(\tau)$ showing the conditions for which the hot spot collapses.

\subsection{Recovery of critical current}

After the film resistance drops to zero, the current increases from its value of $I_{0}$ according to

$$
I=\left(I(0)-I_{0}\right)\left[1-\exp \left(-\tau / \tau_{L R}\right)\right]+I_{0}
$$

where $\tau_{L R} \equiv \mathscr{L} / R_{\mathrm{s}}=250 \mu \mathrm{s}$. The temperature-dependent critical current, $I_{\mathrm{c}}(T)$, has been measured for similar $\mathrm{Nb}_{3} \mathrm{Sn}$ films (Kampwirth et al 1978). In order to compare these as a function of time, it is necessary to know $T(\tau)$. This comes from

$$
\mathrm{d} T / \mathrm{d} \tau=-\left(T-T_{\mathrm{b}}\right) / \tau_{\text {th }}
$$

where $\tau_{\text {th }}$ is the thermal response time of the film-substrate combination: $\tau_{\text {th }}=R_{\mathrm{K}} C_{\mathrm{v}}(T) t$, where $R_{\mathrm{K}} \simeq\left(T_{\mathrm{c}}-T_{\mathrm{b}}\right) / 3 W_{0}$. The factor of three accounts roughly for the ratio of total surface area of the sapphire to the film area (i.e. $2 \times 5.1 \mathrm{~cm} / 3.5 \mathrm{~cm}$ ).

At low temperatures, the current calculated from equation (24) and the integration of equation (25) is always less than about $\frac{2}{3} I_{c}$. However, in the limit of $T \rightarrow T_{c}$, the numerical values of $I$ and $I_{c}$ are very close, indicating that recovery is indeed possible but perhaps marginal. Slight changes in certain parameters could prevent recovery, and such behaviour was indeed far from universal. However, this effect has been seen in a few other $\mathrm{Nb}_{3} \mathrm{Sn}$ samples.

Consequently, the full recovery of current can be understood, and we are left to explain why the hot spot collapses under certain conditions (150 A $<I \leqslant 160 \mathrm{~A}$ ).

\subsection{Collapse of hot spot}

In order to explain the hot spot collapse, a calculation of $L_{n}(\tau)$ and $I(\tau)$ based on the model proposed earlier must be made. The result is in excellent quantitative agreement with the 
experiment. The procedure of the calculation is as follows. Given $L_{\mathrm{n}}$ and $\rho_{\mathrm{n}} J^{2} d$ at time $\tau$, the velocity (as in figure 7) is calculated using equations (1), (6), (12) or (23), appropriately adjusted for finite substrate length. A time interval, $\delta \tau$, is selected which is shorter for high velocities. During this time interval the length $L_{\mathrm{n}}$ changes by $\delta L_{\mathrm{n}}=2 v \delta \tau$ since propagation is from both ends of the hot spot, and the current in the superconductor changes according to

$$
\delta I=\left\{I(0)-\left[1+\left(L_{\mathrm{n}} / L_{\mathrm{s}}\right)\right] I\right\} \delta \tau / \tau_{L R} .
$$

After each time step, the new values of $I+\delta I$ (or $J+\delta J$ ) and $L_{\mathfrak{n}}+\delta L_{\mathfrak{n}}$ are used to calculate a new velocity and the whole procedure repeated. The initial hot spot is created artificially with length $1 \mu \mathrm{m}$. All the parameters of the calculation are measured experimentally (including $C_{v}^{\prime}$ for $\mathrm{Nb}_{3} \mathrm{Sn}$ from Viswanathan and Johnston (1976)) so that the time evolutions of $J$ and $L_{\mathrm{n}}$ are calculated with no adjustable parameter. The results of such a calculation for various values of $I(0)$ are shown in figures 9 and 10 . Figure 9 shows the current through the superconductor as a function of time and is exactly analogous to the switching within the $5 \mathrm{~ms}$ current pulse shown in figures $8(b)$ and $(c)$. There is a sharp decrease (about $10 \mu \mathrm{s}$ ) in the current upon switching $(\tau=0)$, followed by a slow rise over the next $200 \mu \mathrm{s}$. For $I(0) \leqslant 166 \mathrm{~A}$, the current then recovers to its full value with a time constant approximately equal to $\tau_{L R}=250 \mu \mathrm{s}$. However, if $I(0) \geqslant 167 \mathrm{~A}$, the current approaches the steady state value (figure 6) with the small oscillations dying out for larger currents. The agreement with experiment (figure 8 ) is indeed remarkable, considering that there are no adjustable parameters. In figure $8(c)$, heating of the helium bath may be responsible for quenching the recovery after a few cycles. However, when $I(0)$ is increased

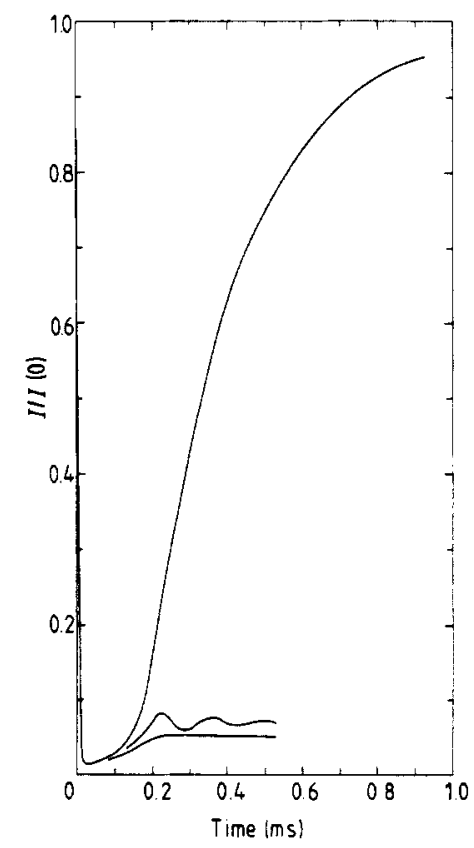

Figure 9. The time dependence of the current through a superconducting film shunted by $6 \mathrm{~m} \Omega$ and $1.5 \mu \mathrm{H}$, calculated using the thermal propagation model represented by equations (1), (12) and (23). There are no adjustable parameters in this attempt to fit the data of figures $8(b)$ and $(c)$. The total current $I(0)$ is 150,175 and $200 \mathrm{~A}$ respectively (top to bottom). 


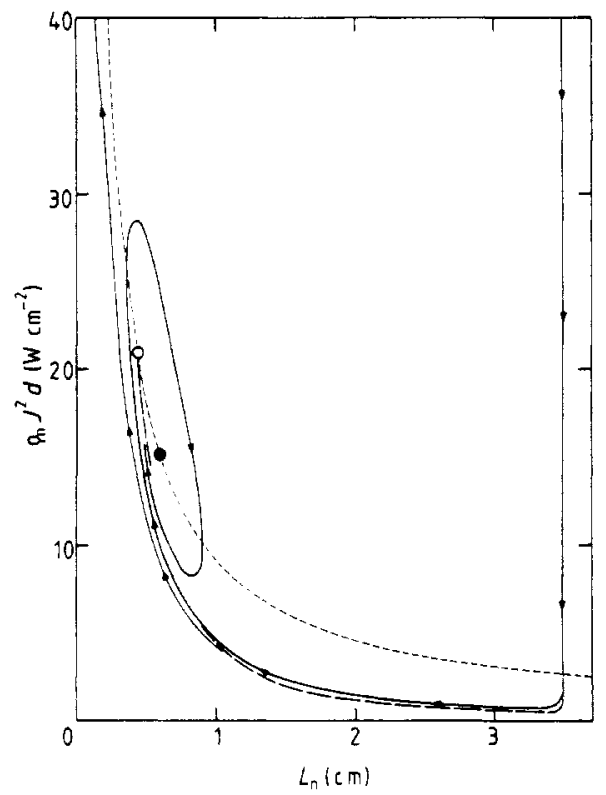

Figure 10. The trajectory of thermal propagation calculated as in figure 9 . The short broken curve is the steady state (zero velocity) solution. The two full curves display the characteristically different behaviours shown in figure 9 and also in the experiments (figure $8)$. In one case $(I(0)=165 \mathrm{~A})$, after dropping below the steady state curve the trajectory never again reaches this curve and the hot spot collapses. In the other case $(I(0)=170 \mathrm{~A})$, the trajectory recrosses the steady state line and spirals into the stable hot spot solution (O). If the inductance is reduced from 1.5 to $0.2 \mu \mathrm{H}$, the trajectory (long broken curve) returns monotonically to the stable hot spot $(O)$ without oscillation, even though $I(0)$ is only $150 \mathrm{~A}$.

somewhat beyond $160 \mathrm{~A}$ in the experiment (not shown in figure 8), the current never recovers and only the steady state solution is reached.

Figure 10 shows the trajectories for $I(0)=165$ and $170 \mathrm{~A}$, as well as the zero-velocity steady state solution (short broken curve). For $165 \mathrm{~A}$, the trajectory narrowly misses the steady state and superconductivity is recovered. When $I(0) \geqslant 167 \mathrm{~A}$ the behaviour is similar to the curve for $170 \mathrm{~A}$ in which the trajectory crosses and recrosses the steady state line eventually arriving at the steady state solution (the full circle which acts as an attractor).

The calculations shown in figures 9 and 10 indicate that the current minimum in the normal state should be about $2 \mathrm{~A}$ whereas the data in figure 8 suggest a minimum value of about $5 \mathrm{~A}$. This discrepancy points out some of the difficulties in the details of such a calculation. In the calculations shown in figures 9 and 10, the BR solutions for propagation in the sapphire substrate were not used, although the velocity was restricted to be less than $s$. If the BR model for propagation in the substrate is included in the calculation, there is a profound effect on the minimum current. This is because the propagation velocity becomes very large and negative for $\rho_{\mathrm{n}} J^{2} d \leqslant 2 W_{0}$ and this effectively prevents the current from falling below about $4.5 \mathrm{~A}$. However, this large negative velocity causes the hot spot to collapse more quickly, and the supercurrent recovers after a much shorter delay time (about $50 \mu$ s compared with $200 \mu \mathrm{s}$ in figure 8). Apparently we are approaching the limit of such a simple model, although the overall predictions are confirmed.

The role of the inductance can be illustrated by a calculation in which $\mathscr{L}$ was reduced 
from 1.5 to $0.2 \mu \mathrm{H}$ (the upper limit measured in experiments shown in figures 2-6). In this case, the calculated trajectory, after dropping to a similar low value of current and $L_{\mathrm{n}}=3.5 \mathrm{~cm}$, moves smoothly to the steady state attractor with no oscillation (even for $I(0)=150 \mathrm{~A}$ ). Of course, for zero inductance it must follow a trajectory similar to the lower one shown in figure 7.

Note that the recovery of superconductivity after commutation into the shunt resistor is an exotic method of current sharing. Usually superconducting wires are imbedded in a copper matrix which provides stability against hot spot propagation in several ways, including heat removal and current sharing. Conventional wisdom then suggests that current sharing lowers the heat dissipation (analogous to $\rho_{\mathrm{n}} J^{2} d$ ) so that the cooling by the copper is sufficient for the hot spot to collapse. Note, however, that copper can also provide eddy-current damping of the flux jumps which presumably initiate hot spot formation (see $\S 6$ ).

\subsection{Conclusions}

In summary, equations (1), (6), (12) and (23), which are represented in figure 7, can be used to predict accurately the behaviour of normal hot spots in superconducting films. The predictions for repetitive switching using no adjustable parameters are particularly striking. However, for thermally conducting substrates, the film must be as wide as the substrate for the one-dimensional calculation to be valid. In addition, the unstable steady state solution on the left of figure 7 gives the relationship of critical current density to a critical hot spot size $L_{\mathrm{nc}}$ which was mentioned at the end of $\S 4$, and will be discussed in greater detail in $\S 6$.

\section{Critical current stability}

The discussion of $\S 4$ suggested that a great deal of the critical current stability in high $\rho_{\mathrm{n}} J^{2} d$ superconducting films is provided by efficient heat removal through the substrate. In this section, additional information in support of this conclusion is reviewed, including experiments showing self-healing of hot spots. The concept of critical hot spot size is more fully developed, along with a discussion of the conclusions.

It is not surprising that thermal effects are important to the stability of high normalstate-resistivity superconductors carrying large current densities, since for large enough $\rho_{\mathrm{n}} J^{2}$, any disturbance must generate enough heat to quench the superconductor. The previous discussion, particularly in $\S 4$ underlines this problem. An important additional consideration is the probability of occurrence of a disturbance (say a thermally activated flux jump) which is large enough to propagate throughout the superconducting film. On this latter point there seems to be only limited empirical data (see below) which is particular to each material and fabrication procedure (i.e. metallurgy). However, on the question of whether a disturbance will propagate, it is felt that the model presented previously and the ensuing discussion provides a basis for understanding and prediction.

\subsection{Stabilisation by heat removal}

The role of heat removal in stabilising high values of $J_{c}$ was studied (Kampwirth and Gray 1981 ) in $\mathrm{NbN}$ films, since these high $\rho_{\mathrm{n}} J^{2}$ films could be made on both glass (thermally 
insulating) and sapphire (thermally conducting) substrates. Using glass substrates, the measured $J_{c}$ decreased for increasing film width and for relatively fast current rise time (about $1 \mathrm{~ms}$ ). This seemed to indicate a stability problem (rather than metallurgical differences): a fast current rise leads to a shorter time for cooling between successive flux jumps; the flux, concentrated at the edges (Migliori et al 1977) of the films, must jump greater distances for wider films, thereby dissipating more heat.

That it was a stability problem was verified by depositing a $6-10 \mu \mathrm{m}$ film of high-purity copper directly onto the $\mathrm{NbN}$ films on glass substrates. As a result the decreases in $J_{\mathrm{c}}$ due to width and fast current rise were eliminated. However, it is well known that in addition to heat removal, copper can also stabilise a superconductor by eddy-current damping of flux jumps and by current sharing. In order to determine which stabilising effect dominated, the same films were deposited onto sapphire substrates which can act only as heat sinks. $\mathrm{NbN}$ on sapphire substrates reproduced the values of $J_{c}$ for copper overcoated $\mathrm{NbN}$ on glass, including the independence of $J_{c}$ on width and current rise time. As a final check, the $\mathrm{NbN}$ samples on sapphire were also overcoated with copper, resulting in no substantial additional improvement in $J_{\mathrm{c}}$ (DC or fast current rise).

Thus it can be concluded that heat removal is an important determining factor in $J_{c}$ for these films and the model expressed in equation (22) is relevant to our understanding of limitations and possible improvements of $J_{\mathfrak{c}}$. For example, good thermal coupling of the film to the substrate (i.e. large $W_{0}^{\prime}$ ) can increase $J_{\mathrm{c}}$, all other things being the same. Improvements in $W_{0}^{\prime}$ can result from good film adherence and by matching the acoustic impedances of the film and substrate (see Kaplan 1979). Unfortunately, it is unlikely that the thermal conductivity $K^{\prime}$ can be increased for high normal resistivity films (e.g. for switch applications (Gray and Fowler 1978)), since $K^{\prime} \propto 1 / \rho_{\mathrm{n}}$.

\subsection{Self-healing hot spots}

During the course of experiments to measure the critical current density $J_{c}$ in various niobium-based superconductors on sapphire substrates, a rather surprising effect was seen. With the current increasing slowly but steadily (taking several minutes to reach $J_{\mathrm{c}}$ ), the samples often became resistive for short periods of time (i.e. voltage pulses of duration less than $1 \mathrm{~ms}$ ) after which they recovered superconductivity fully. There is an important difference between this rather general behaviour and the recovery reported at the end of $\S 5$. In the present case there is no commutation of the current into the shunt resistor. Thus the impressive feature of this recovery was that the value of $\rho_{\mathrm{n}} J^{2} d$ was as large as $1.5 \times 10^{4} \mathrm{~W} \mathrm{~cm}^{-2}$, i.e. much greater than $W_{0}^{\prime} \sim 125 \mathrm{~W} \mathrm{~cm}^{-2}$, the permissible heat flux across the film-substrate boundary for a temperature rise to $T_{c}$. A second important fact is that this behaviour has never been seen in samples on glass substrates.

These voltage pulses can arise from a short length of superconductor becoming completely normal as a result of a highly localised flux jump-along a grain boundary or in a microscopic region of weak superconductivity. It is interesting to estimate the length of the normal region to compare it with the critical length for the appropriate value of $\rho_{\mathrm{n}} J^{2} d$ using equation (21). In the cases studied, the measured voltage indicated normal regions varying from $40 \AA$ for $\mathrm{Nb}_{3} \mathrm{Ge}$ to $60-100 \AA$ for $\mathrm{NbN}$. These are considerably less than the critical values of $L_{n}$ obtained from equation (21) which are of the order 600-1000 $\AA$, and thus the model of $\S 4$ would predict recovery. It should also be pointed out that for glass substrates, the critical values of $L_{n}$ are about 60-100 $\AA$ (see figure 6) so that recovery would be much less likely.

However, the measurements must be considered only as a lower bound on the 
magnitude of the voltage since no transient recorder was available and the intermittent pulses could only be measured with an amplifier and $x-y$ recorder having a restricted band width. An upper bound of about $1 \mu \mathrm{m}$ for $L_{\mathrm{n}}$ can be estimated from the lack of any observable current commutation into the shunt.

It is interesting to consider whether these normal region sizes correspond to characteristic length scales in the films. For example, the superconducting coherence length $\xi$ is of the order of $50-100 \AA$, the magnetic field penetration depth $\lambda$ is of the order of $1000-2000 \AA$ and the grain sizes are perhaps $1000-5000 \AA$. When magnetic flux moves, the dissipation is mainly confined to the normal core (Bardeen and Stephen 1965) of approximate radius $\xi$. These temporary breakdowns are probably initiated by flux jumps of the self-field of the current. If this occurs along a single weak path (e.g. a grain boundary) a normal region of approximate size $2 \xi \sim 100-200 \AA$ is feasible. Such a size would fall between the experimental limits as well as between the critical values for glass and sapphire substrates from equation (21).

\subsection{Niobium germanium}

The case of $\mathrm{Nb}_{3} \mathrm{Ge}$ is particularly interesting because the voltage pulses can be definitely associated with a second metallurgical phase of a weaker superconductor. High $T_{\mathrm{c}}$ (about $22 \mathrm{~K}$ ) samples of $\mathrm{Nb}_{3} \mathrm{Ge}$ require the metastable $\mathrm{A}-15$ structure to be thermally quenched by condensation onto substrates heated to $750-800^{\circ} \mathrm{C}$. This has been accomplished by chemical vapour deposition (Thompson et al 1977, Braginski et al 1978) and by sputtering as in the case of our films (Kampwirth 1979). Other methods lead to a germanium-deficient A-15 phase (Matthias et al 1965) of low $T_{\mathrm{c}}$ (about $6 \mathrm{~K}$ ), which is sometimes found as an impurity in films which otherwise show the proper A-15 structure. Susceptibility measurements verified that this impurity phase was present in the $\mathrm{Nb}_{3} \mathrm{Ge}$ films exhibiting voltage pulses.

Convincing evidence that the voltage pulses are related to the second phase results from the temperature and magnetic field dependence of $J_{c}$. It was noted that a field of a few $\mathrm{kG}$, applied to these $\mathrm{Nb}_{3} \mathrm{Ge}$ films at $4.2 \mathrm{~K}$, caused the voltage pulses to disappear but, more surprisingly, resulted in an increase of $J_{c}$ (see figure 11). This behaviour is completely opposite to ordinary superconductors; however, for fields greater than about $2 \mathrm{kG}$ the $J_{\mathrm{c}}$ decreased in the normal way.

The connection with the germanium-deficient impurity is seen more clearly in the temperature dependence shown in figure 12. In zero magnetic field, the $J_{\mathrm{c}}$ at $4.2 \mathrm{~K}$ increases dramatically by more than a factor of two at about $6 \mathrm{~K}$, the $T_{\mathrm{c}}$ of this impurity phase. Similarly, the voltage pulses cease, indicating that the flux jumps creating the pulses occur in the impurity regions which exhibit weak superconductivity. Above $6 \mathrm{~K}$, these regions are in the normal state so the supercurrent avoids them and no flux jumps occur. Evidently a field of $1-2 \mathrm{kG}$ is likewise sufficient to drive the impurity phase normal and stabilise the flux jumps. These are demonstrated by the data at $7.0 \mathrm{~K}$ in figure 11 and $2.5 \mathrm{kG}$ in figure 12 .

\subsection{Niobium nitride and niobium tin}

For the cases of $\mathrm{NbN}$ and $\mathrm{Nb}_{3} \mathrm{Sn}$, a magnetic field is also observed to quench the voltage pulses; however, a larger critical current does not result. Presumably at low temperatures and zero field, the weak regions in these superconductors are not as effective at lowering 


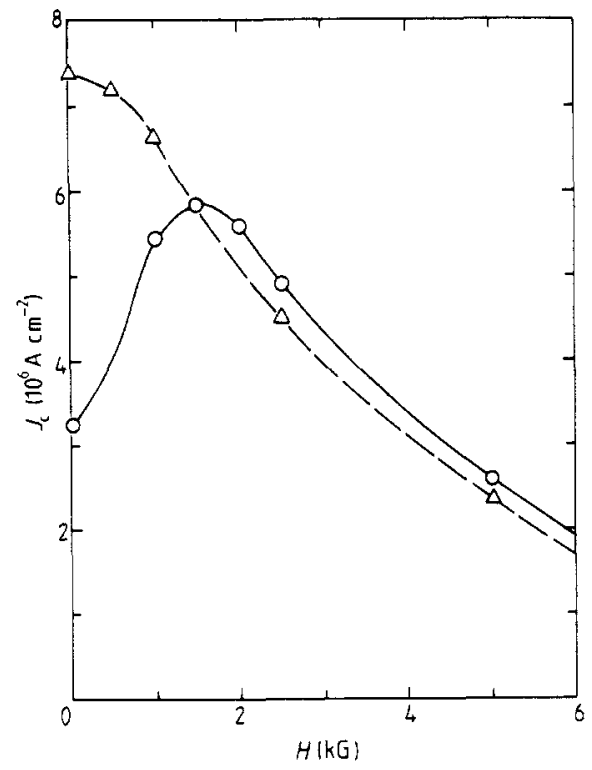

Figure 11. The critical current density of a $\mathrm{Nb}_{3} \mathrm{Ge}$ film (NG82R) as a function of applied magnetic field at $4.2 \mathrm{~K}(O)$ and $7.0 \mathrm{~K}(\Delta)$ showing the effect of a low $T_{\mathrm{c}}$ second phase impurity.

$J_{c}$, and the field necessary to quench the flux jumps causes a somewhat larger decrease in $J_{\mathrm{c}}$.

There is no evidence for other phases in the $\mathrm{NbN}$ or $\mathrm{Nb}_{3} \mathrm{Sn}$ films, although weak spots

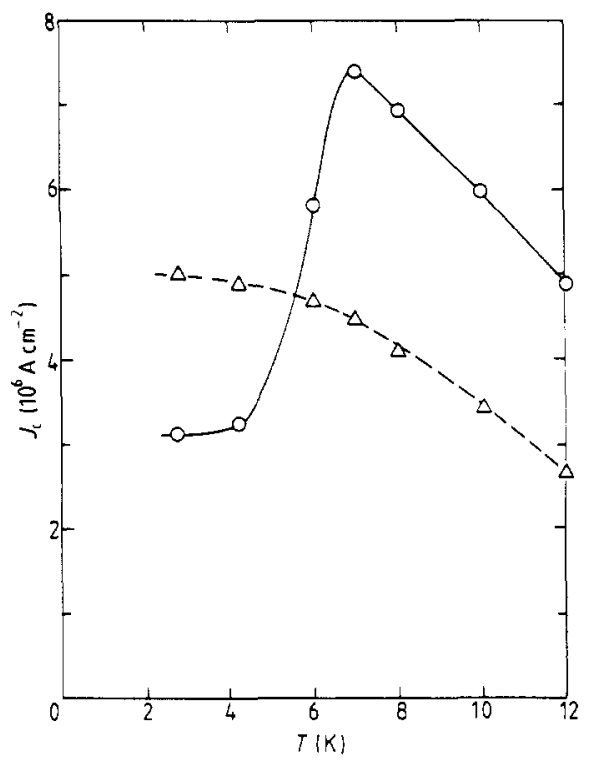

Figure 12. The critical current density for the same sample as figure 11, plotted as a function of temperature for zero magnetic field $(O)$ and for $2.5 \mathrm{kG}(\triangle)$. As the second phase becomes superconducting below about $6 \mathrm{~K}$, flux jumps can occur which propagate thermally and quench superconductivity. In a higher field, these second phase impurities are driven normal and $J_{c}$ behaves normally. 
must exist. In $\mathrm{Nb}_{3} \mathrm{Sn}$ one might speculate that they are grain boundaries, perhaps with an excess of impurities or structural imperfections. On the other hand, previous experiments (Kampwirth and Gray 1981, Wolf and Gubser 1979) on NbN have shown this highresistivity material to behave like a granular material in which Josephson coupling between grains supports the supercurrent. Regions with the weakest grain-to-grain coupling would be highly susceptible to flux jumps. In both of the above cases one can speculate that the main effect of the magnetic field is to drop $J_{c}$ below the critical value for the self-healing flux jumps.

\subsection{Discussion}

Apparently one cannot rule out the possibility that the flux jumps occur over a much longer section of superconductor which is not driven completely normal (i.e. $\rho_{\mathrm{n}}>\rho>0$ ). Such regions will also result in permanent breakdown if the hot spot propagates, and the conditions for recovery (as opposed to thermal propagation) contained in equation (21), imply exactly the same limits on the magnitude of the voltage across the region $V^{\prime}$, i.e.

$$
V^{\prime}<2 W_{0}^{\prime} \eta_{\mathrm{f}} / J d
$$

since the effective resistivity $\rho$ (less than $\rho_{n}$ ) cancels out. Of course if the power per unit area $\rho J^{2} d$ is less than $2 W_{0}^{\prime}$, then recovery can occur for a much larger range of voltage:

$$
V^{\prime}<4 W_{0}[\eta \tanh (L / 2 \eta)] / J d .
$$

This situation, in which the breakdown is not to the normal state $\left(T>T_{\mathrm{c}}\right)$, is quite different since the temperature rise may be significantly less than to $T_{c}$. Therefore immediately after the flux jump occurs, a zero or very low resistance state can exist which guarantees recovery.

\section{Conclusions}

In summarising the previous sections, the most important conclusion about thermal propagation is the distinction of two regimes depending on the relationship of $L_{\mathrm{n}}$ to $\eta^{*}$, the velocity-dependent dynamical thermal healing length (figure 7 ). For $L_{\mathrm{n}} \gg \eta^{*}$ the BR model has been verified quantitatively. In the opposite case, $L_{n} \ll \eta^{*}$, a new energy balance model is proposed which agrees semi-quantitatively with experiment.

For superconducting films on high thermal conductivity substrates one must differentiate between two additional cases: at low power $\left(\rho_{\mathrm{n}} J^{2} d<W_{0}^{\prime}\right)$, the film and substrate are well coupled and propagation is controlled by the more massive substrate; however, for higher power and small hot spot length, the film can become decoupled from the substrate and propagation occurs in the film alone. These four regimes (two for insulating substrates) also manifest themselves in the stable steady state solutions (figure $6)$.

The general picture which emerges from these considerations is quite helpful to our understanding of a wide variety of problems concerning thin superconducting films. These include critical current stabilisation, protection against fusing after an accidental quench, switching by thermal propagation, repetitive switching (recovery after current commutation) and self-healing of short hot spots without current commutation where the power density is very high $\left(\rho_{\mathrm{n}} J^{2} d \gg W_{0}^{\prime}\right)$. 
Finally the role of low $T_{\mathrm{c}}$ regions in the critical current of $\mathrm{Nb}_{3} \mathrm{Ge}$ films is confirmed, and is consistent with the above general picture.

\section{Acknowledgments}

The basis of the work reported in this paper has been accumulated over many years. The authors would like to acknowledge the USDOE, Division of Electrical Energy Systems for their support through a collaboration (Contract ET-78-C-01-3015) with Gould-Brown Boveri (see Gray et al 1980). Additional continuing support has come from the USDOE, Division of Basic Energy Sciences. We would like to acknowledge discussions with Professor $\mathrm{H}$ Weinstock and Professor John Clem and the technical assistance of $\mathrm{Mr}$ Zenon Sungaila.

\section{References}

Bardeen J and Stephen M J 1965 Phys. Rev. 140 A 1197

Braginski A I, Rowland G W, Daniel M R, Sauthanam A T and Guardipee K W 1978 J. Appl. Phys. 49736

Bremer J W and Newhouse V L 1958 Phys. Rev. Lett. 1505

Broom R F and Rhoderick E H 1960 Br.J. Appl. Phys. 11292

Cody G D and Cohen R W 1964 Rev. Mod. Phys. 36121

Gray K E 1976 J. Low Temp. Phys. 23679

Gray K Eand Fowler D E 1978 J. Appl. Phys. 492546

Gray K E, Kampwirth R T and Zasadzinski J F 1980 FINAL Report: Superconducting Fault Current Limiter USDOE Contract ET-78-01-3015 ch. 4

Gray K E, Lenihan T and Tarczon J 1979 IEEE Trans. Magn. MAG-15 175

Kampwirth R T 1979 IEEE Trans. Magn. MAG-15 502

Kampwirth R T and Gray K E 1981 IEEE Trans. Magn. MAG-17 565

Kampwirth R T, Hafstrom J W and Wu C T 1977 IEEE Trans. Magn. MAG-13 315

Kampwirth R T, Wu C T and Hafstrom J W 1978 Adv. Cryogenic Eng. 24465

Kaplan S B 1979 J. Low Temp. Phys. 37343

Kittel C 1968 Introduction to Solid State Physics 3rd edn (New York: Wiley) p 186

Matthias B T, Geballe T H, Willens R H, Corenzwit E and Hull G W Jr 1965 Phys. Rev. 139 A1501

Migliori A, Taylor R D and Bartlett R J 1977 IEEE Trans. Magn. MAG-15 198

Neeper D A and Dillinger J R 1964 Phys. Rev. 135 A1028

Newhouse V L 1964 Applied Superconductivity (New York: Wiley)

Skocpol W J, Beasley M R and Tinkham M 1974 J. Appl. Phys. 454054

Steward W G 1978 Int. J. Heat Mass Transfer 21863

Thompson J D, Maley M P and Newkirk L R 1977 Appl. Phys. Lett. 30190

Touloukian Y S 1970 Thermophysical Properties of Matter (New York: Plenum) vol. 1, 4

Ulbricht A 1979 IEEE Trans. Magn. MAG-15 172

Viswanathan R and Johnston D C 1976 J. Low Temp. Phys. 251

Wilson M N and Iwasa Y 1978 Cryogenics 1817

Wipf S L 1979 IEEE Trans. Magn. MAG-17 379

Wipf S L and Martinelli A P 1973 Proc. IEEE Conf. on Applied Superconductivity, 1972 CH0682-5-TABSC p 331

Wolf S A and Gubser D U 1979 Solid State Commun. 32449 